\title{
Post-acute COVID-19 syndrome
}

\author{
Ani Nalbandian ${ }^{1,24}$, Kartik Sehgal ${ }^{2,3,4,24 \bowtie, ~ A a k r i t i ~ G u p t a ~}{ }^{1,5,6}$, Mahesh V. Madhavan ${ }^{1,5}$, \\ Claire McGroder ${ }^{7}{ }^{7}$, Jacob S. Stevens ${ }^{8}$, Joshua R. Cook ${ }^{10}{ }^{9}$, Anna S. Nordvig ${ }^{10}{ }^{10}$, Daniel Shalev ${ }^{11}$, \\ Tejasav S. Sehrawat ${ }^{12}$, Neha Ahluwalia ${ }^{13}$, Behnood Bikdeli ${ }^{4,5,6,14}$, Donald Dietz ${ }^{15}$, \\ Caroline Der-Nigoghossian ${ }^{16}$, Nadia Liyanage-Don ${ }^{17}$, Gregg F. Rosner ${ }^{1}$, Elana J. Bernstein ${ }^{18}{ }^{18}$, \\ Sumit Mohan ${ }^{8}{ }^{8}$, Akinpelumi A. Beckley ${ }^{19}$, David S. Seres ${ }^{20}$, Toni K. Choueiri ${ }^{2,3,4}$, Nir Uriel', \\ John C. Ausiello9, Domenico Accili ${ }^{9}$, Daniel E. Freedberg ${ }^{21}$, Matthew Baldwin ${ }^{7}{ }^{7}$, Allan Schwartz', \\ Daniel Brodie ${ }^{7}{ }^{7}$, Christine Kim Garcia7, Mitchell S. V. Elkind (10,22, Jean M. Connors ${ }^{4,23}$, \\ John P. Bilezikian ${ }^{9}$, Donald W. Landry ${ }^{8}$ and Elaine Y. Wan ${ }^{1 凶}$
}

Severe acute respiratory syndrome coronavirus 2 (SARS-CoV-2) is the pathogen responsible for the coronavirus disease 2019 (COVID-19) pandemic, which has resulted in global healthcare crises and strained health resources. As the population of patients recovering from COVID-19 grows, it is paramount to establish an understanding of the healthcare issues surrounding them. COVID-19 is now recognized as a multi-organ disease with a broad spectrum of manifestations. Similarly to post-acute viral syndromes described in survivors of other virulent coronavirus epidemics, there are increasing reports of persistent and prolonged effects after acute COVID-19. Patient advocacy groups, many members of which identify themselves as long haulers, have helped contribute to the recognition of post-acute COVID-19, a syndrome characterized by persistent symptoms and/or delayed or long-term complications beyond 4 weeks from the onset of symptoms. Here, we provide a comprehensive review of the current literature on post-acute COVID-19, its pathophysiology and its organ-specific sequelae. Finally, we discuss relevant considerations for the multidisciplinary care of COVID-19 survivors and propose a framework for the identification of those at high risk for post-acute COVID-19 and their coordinated management through dedicated COVID-19 clinics.

S evere acute respiratory syndrome coronavirus 2 (SARS-CoV-2), the pathogen responsible for coronavirus disease 2019 (COVID-19), has caused morbidity and mortality at an unprecedented scale globally ${ }^{1}$. Scientific and clinical evidence is evolving on the subacute and long-term effects of COVID-19, which can affect multiple organ systems ${ }^{2}$. Early reports suggest residual effects of SARS-CoV-2 infection, such as fatigue, dyspnea, chest pain, cognitive disturbances, arthralgia and decline in quality of life $e^{3-5}$. Cellular damage, a robust innate immune response with inflammatory cytokine production, and a pro-coagulant state induced by SARS-CoV-2 infection may contribute to these sequela $^{6-8}$. Survivors of previous coronavirus infections, including the SARS epidemic of 2003 and the Middle East respiratory syndrome (MERS) outbreak of 2012, have demonstrated a similar constellation of persistent symptoms, reinforcing concern for clinically significant sequelae of COVID-19 (refs. ${ }^{9-15}$ ).

Systematic study of sequelae after recovery from acute COVID19 is needed to develop an evidence-based multidisciplinary team

\footnotetext{
'Division of Cardiology, Department of Medicine, Vagelos College of Physicians and Surgeons, New York-Presbyterian/Columbia University Irving Medical Center, New York, New York, USA. '² Department of Medical Oncology, Dana-Farber Cancer Institute, Boston, Massachusetts, USA. ${ }^{3}$ Department of Medicine, Brigham and Women's Hospital, Boston, Massachusetts, USA. ${ }^{4}$ Harvard Medical School, Boston, Massachusetts, USA. ${ }^{5} \mathrm{Clinical}$ Trials Center, Cardiovascular Research Foundation, New York, New York, USA. ${ }^{6}$ Center for Outcomes Research and Evaluation, Yale New Haven Hospital, New Haven, Connecticut, USA. 'Division of Pulmonary, Allergy and Critical Care Medicine, Department of Medicine, Vagelos College of Physicians and Surgeons, New York-Presbyterian/Columbia University Irving Medical Center, New York, New York, USA. ${ }^{8}$ Division of Nephrology, Department of Medicine, Vagelos College of Physicians and Surgeons, New York-Presbyterian/Columbia University Irving Medical Center, New York, New York, USA. ${ }^{9}$ Division of Endocrinology, Department of Medicine, Vagelos College of Physicians and Surgeons, New York-Presbyterian/Columbia University Irving Medical Center, New York, New York, USA. ${ }^{10}$ Department of Neurology, Vagelos College of Physicians and Surgeons, New York-Presbyterian/Columbia University Irving Medical Center, New York, New York, USA. "Department of Psychiatry, Vagelos College of Physicians and Surgeons, New York-Presbyterian/Columbia University Irving Medical Center, and New York State Psychiatric Institute, New York, New York, USA. ${ }^{2}$ Division of Gastroenterology and Hepatology, Department of Medicine, Mayo Clinic, Rochester, Minnesota, USA. ${ }^{13}$ Division of Cardiology, Department of Pediatrics, Icahn School of Medicine at Mount Sinai, New York, New York, USA. ${ }^{14}$ Cardiovascular Division, Brigham and Women's Hospital, Boston, Massachusetts, USA. ${ }^{15}$ Division of Infectious Diseases, Department of Medicine, Vagelos College of Physicians and Surgeons, New York-Presbyterian/Columbia University Irving Medical Center, New York, New York, USA. ${ }^{16}$ Clinical Pharmacy, New York-Presbyterian Hospital/Columbia University Irving Medical Center, New York, New York, USA. ${ }^{17}$ Department of Medicine, Vagelos College of Physicians and Surgeons, New York-Presbyterian/Columbia University Irving Medical Center, New York, New York, USA. ${ }^{18}$ Division of Rheumatology, Department of Medicine, Vagelos College of Physicians and Surgeons, New York-Presbyterian/Columbia University Irving Medical Center, New York, New York, USA. ${ }^{19}$ Department of Rehabilitation and Regenerative Medicine, New York-Presbyterian/Columbia University Irving Medical Center, New York, New York, USA. ${ }^{20}$ Institute of Human Nutrition and Division of Preventive Medicine and Nutrition, Department of Medicine, Vagelos College of Physicians and Surgeons, New York-Presbyterian/Columbia University Irving Medical Center, New York, New York, USA. ${ }^{21}$ Division of Digestive and Liver Diseases, Department of Medicine, Vagelos College of Physicians and Surgeons, New York-Presbyterian/Columbia University Irving Medical Center, New York, New York, USA. 22 Department of Epidemiology, Mailman School of Public Health, Columbia University, New York, New York, USA. ${ }^{23}$ Division of Hematology, Brigham and Women's Hospital, Boston, Massachusetts, USA. ${ }^{24}$ These authors contributed equally: Ani Nalbandian, Kartik Sehgal.凶e-mail: Kartik_sehgal@dfci.harvard.edu; eyw2003@cumc.columbia.edu
} 


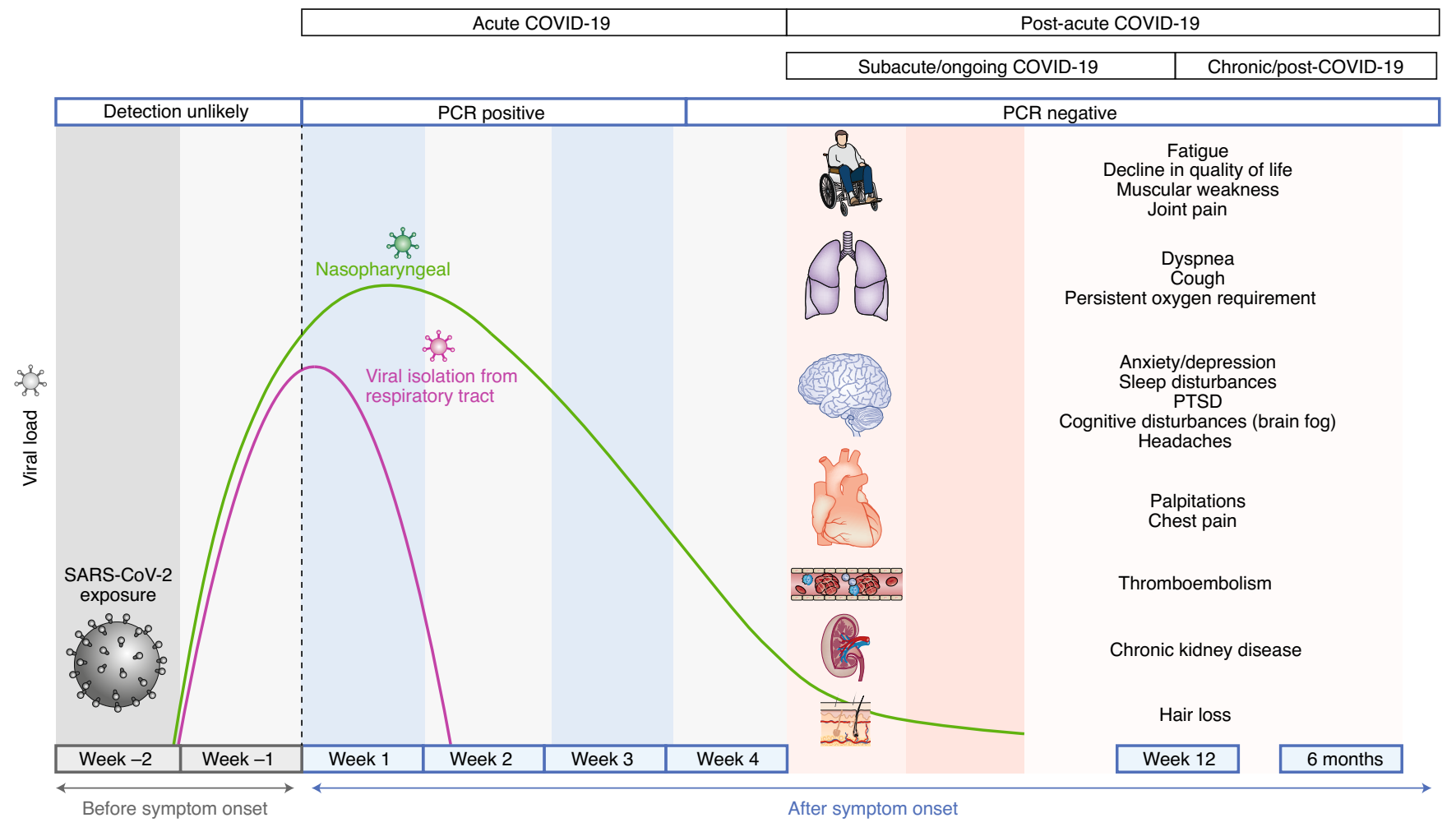

Fig. 1 | Timeline of post-acute COVID-19. Acute COVID-19 usually lasts until 4 weeks from the onset of symptoms, beyond which replication-competent SARS-CoV-2 has not been isolated. Post-acute COVID-19 is defined as persistent symptoms and/or delayed or long-term complications beyond 4 weeks from the onset of symptoms. The common symptoms observed in post-acute COVID-19 are summarized.

approach for caring for these patients, and to inform research priorities. A comprehensive understanding of patient care needs beyond the acute phase will help in the development of infrastructure for COVID-19 clinics that will be equipped to provide integrated multispecialty care in the outpatient setting. While the definition of the post-acute COVID-19 timeline is evolving, it has been suggested to include persistence of symptoms or development of sequelae beyond 3 or 4 weeks from the onset of acute symptoms of COVID19 (refs. ${ }^{16,17}$ ), as replication-competent SARS-CoV-2 has not been isolated after 3 weeks ${ }^{18}$. For the purpose of this review, we defined post-acute COVID-19 as persistent symptoms and/or delayed or long-term complications of SARS-CoV-2 infection beyond 4 weeks from the onset of symptoms (Fig. 1). Based on recent literature, it is further divided into two categories: (1) subacute or ongoing symptomatic COVID-19, which includes symptoms and abnormalities present from 4-12 weeks beyond acute COVID-19; and (2) chronic or post-COVID-19 syndrome, which includes symptoms and abnormalities persisting or present beyond 12 weeks of the onset of acute COVID-19 and not attributable to alternative diagnoses $^{17,19}$. Herein, we summarize the epidemiology and organ-specific sequelae of post-acute COVID-19 and address management considerations for the interdisciplinary comprehensive care of these patients in COVID-19 clinics (Box 1 and Fig. 2).

\section{Epidemiology}

Early reports have now emerged on post-acute infectious consequences of COVID-19, with studies from the United States, Europe and China reporting outcomes for those who survived hospitalization for acute COVID-19. The findings from studies reporting outcomes in subacute/ongoing symptomatic COVID-19 and chronic/ post-COVID-19 syndrome are summarized in Table 1.

An observational cohort study from 38 hospitals in Michigan, United States evaluated the outcomes of 1,250 patients discharged alive at $60 \mathrm{~d}$ by utilizing medical record abstraction and telephone surveys (hereby referred to as the post-acute COVID-19 US study) ${ }^{20}$. During the study period, $6.7 \%$ of patients died, while $15.1 \%$ of patients required re-admission. Of 488 patients who completed the telephone survey in this study, $32.6 \%$ of patients reported persistent symptoms, including $18.9 \%$ with new or worsened symptoms. Dyspnea while walking up the stairs $(22.9 \%)$ was most commonly reported, while other symptoms included cough (15.4\%) and persistent loss of taste and/or smell (13.1\%).

Similar findings were reported from studies in Europe. A post-acute outpatient service established in Italy (hereby referred to as the post-acute COVID-19 Italian study) ${ }^{3}$ reported persistence of symptoms in $87.4 \%$ of 143 patients discharged from hospital who recovered from acute COVID-19 at a mean follow-up of $60 \mathrm{~d}$ from the onset of the first symptom. Fatigue (53.1\%), dyspnea (43.4\%), joint pain $(27.3 \%)$ and chest pain $(21.7 \%)$ were the most commonly reported symptoms, with $55 \%$ of patients continuing to experience three or more symptoms. A decline in quality of life, as measured by the EuroQol visual analog scale, was noted in $44.1 \%$ of patients in this study. A study focused on 150 survivors of non-critical COVID-19 from France similarly reported persistence of symptoms in two-thirds of individuals at $60 \mathrm{~d}$ follow-up, with one-third reporting feeling worse than at the onset of acute COVID-19 (ref. $\left.{ }^{21}\right)$. Other studies, including in-person prospective follow-up studies of 110 survivors in the United Kingdom at 8-12 weeks after hospital admission ${ }^{22}$ and 277 survivors in Spain at 10-14 weeks after disease onset ${ }^{23}$, as well as survey studies of 100 COVID-19 survivors in the United Kingdom at $4-8$ weeks post-discharge $e^{24}$, 183 individuals in the United States at $35 \mathrm{~d}$ post-discharge $\mathrm{e}^{25}$ and 120 patients discharged from hospital in France, at $100 \mathrm{~d}$ following admission $^{26}$, reported similar findings. Fatigue, dyspnea and psychological distress, such as post-traumatic stress disorder (PTSD), anxiety, depression and concentration and sleep abnormalities, 


\section{Box 1 | Summary of post-acute COVID-19 by organ system}

\section{Pulmonary}

- Dyspnea, decreased exercise capacity and hypoxia are commonly persistent symptoms and signs

- Reduced diffusion capacity, restrictive pulmonary physiology, and ground-glass opacities and fibrotic changes on imaging have been noted at follow-up of COVID-19 survivors

- Assessment of progression or recovery of pulmonary disease and function may include home pulse oximetry, 6MWTs, PFTs, high-resolution computed tomography of the chest and computed tomography pulmonary angiogram as clinically appropriate

\section{Hematologic}

- Thromboembolic events have been noted to be $<5 \%$ in post-acute COVID-19 in retrospective studies

- The duration of the hyperinflammatory state induced by infection with SARS-CoV-2 is unknown

- Direct oral anticoagulants and low-molecular-weight heparin may be considered for extended thromboprophylaxis after risk-benefit discussion in patients with predisposing risk factors for immobility, persistently elevated D-dimer levels (greater than twice the upper limit of normal) and other high-risk comorbidities such as cancer

\section{Cardiovascular}

- Persistent symptoms may include palpitations, dyspnea and chest pain

- Long-term sequelae may include increased cardiometabolic demand, myocardial fibrosis or scarring (detectable via cardiac MRI), arrhythmias, tachycardia and autonomic dysfunction

- Patients with cardiovascular complications during acute infection or those experiencing persistent cardiac symptoms may be monitored with serial clinical, echocardiogram and electrocardiogram follow-up

\section{Neuropsychiatric}

- Persistent abnormalities may include fatigue, myalgia, headache, dysautonomia and cognitive impairment (brain fog)

- Anxiety, depression, sleep disturbances and PTSD have been reported in $30-40 \%$ of COVID-19 survivors, similar to survivors of other pathogenic coronaviruses

- The pathophysiology of neuropsychiatric complications is mechanistically diverse and entails immune dysregulation, inflammation, microvascular thrombosis, iatrogenic effects of medications and psychosocial impacts of infection

\section{Renal}

- Resolution of AKI during acute COVID-19 occurs in the majority of patients; however, reduced eGFR has been reported at 6 months follow-up

- COVAN may be the predominant pattern of renal injury in individuals of African descent

- COVID-19 survivors with persistent impaired renal function may benefit from early and close follow-up in AKI survivor clinics

\section{Endocrine}

- Endocrine sequelae may include new or worsening control of existing diabetes mellitus, subacute thyroiditis and bone demineralization

- Patients with newly diagnosed diabetes in the absence of traditional risk factors for type 2 diabetes, suspected hypothalamic-pituitary-adrenal axis suppression or hyperthyroidism should undergo the appropriate laboratory testing and should be referred to endocrinology

\section{Gastrointestinal and hepatobiliary}

- Prolonged viral fecal shedding can occur in COVID-19 even after negative nasopharyngeal swab testing

- COVID-19 has the potential to alter the gut microbiome, including enrichment of opportunistic organisms and depletion of beneficial commensals

\section{Dermatologic}

- Hair loss is the predominant symptom and has been reported in approximately $20 \%$ of COVID-19 survivors

\section{MIS-C}

- Diagnostic criteria: <21 years old with fever, elevated inflammatory markers, multiple organ dysfunction, current or recent SARS-CoV-2 infection and exclusion of other plausible diagnoses

- Typically affects children $>7$ years and disproportionately of African, Afro-Caribbean or Hispanic origin

- Cardiovascular (coronary artery aneurysm) and neurologic (headache, encephalopathy, stroke and seizure) complications can occur were noted in approximately $30 \%$ or more study participants at the time of follow-up.

In a prospective cohort study from Wuhan, China, long-term consequences of acute COVID-19 were evaluated by comprehensive in-person evaluation of 1,733 patients at 6 months from symptom onset (hereby referred to as the post-acute COVID-19 Chinese study) $)^{5}$. The study utilized survey questionnaires, physical examination, 6-min walk tests (6MWT) and blood tests and, in selected cases, pulmonary function tests (PFTs), high-resolution computed tomography of the chest and ultrasonography to evaluate post-acute COVID-19 end organ injury. A majority of the patients (76\%) reported at least one symptom. Similar to other studies, fatigue/muscular weakness was the most commonly reported symptom (63\%), followed by sleep difficulties (26\%) and anxiety/ depression (23\%).

These studies provide early evidence to aid the identification of people at high risk for post-acute COVID-19. The severity of illness during acute COVID-19 (measured, for example, by admission to an intensive care unit (ICU) and/or requirement for non-invasive and/ or invasive mechanical ventilation) has been significantly associated with the presence or persistence of symptoms (such as dyspnea, fatigue/muscular weakness and PTSD), reduction in health-related quality of life scores, pulmonary function abnormalities and radiographic abnormalities in the post-acute COVID-19 setting ${ }^{5,22,24}$. Furthermore, Halpin et al. ${ }^{24}$ reported additional associations between pre-existing respiratory disease, higher body mass index, older age and Black, Asian and minority ethnic (BAME) and dyspnea at 4-8 weeks follow-up. The post-acute COVID-19 Chinese study also suggested sex differences, with women more likely to experience fatigue and anxiety/depression at 6 months follow-up ${ }^{5}$, similar to SARS survivors ${ }^{15}$. While other comorbidities, such as diabetes, obesity, chronic cardiovascular or kidney disease, cancer and organ transplantation, are well-recognized determinants of increased severity and mortality related to acute COVID-19 (refs. ${ }^{2,27}$ ), 


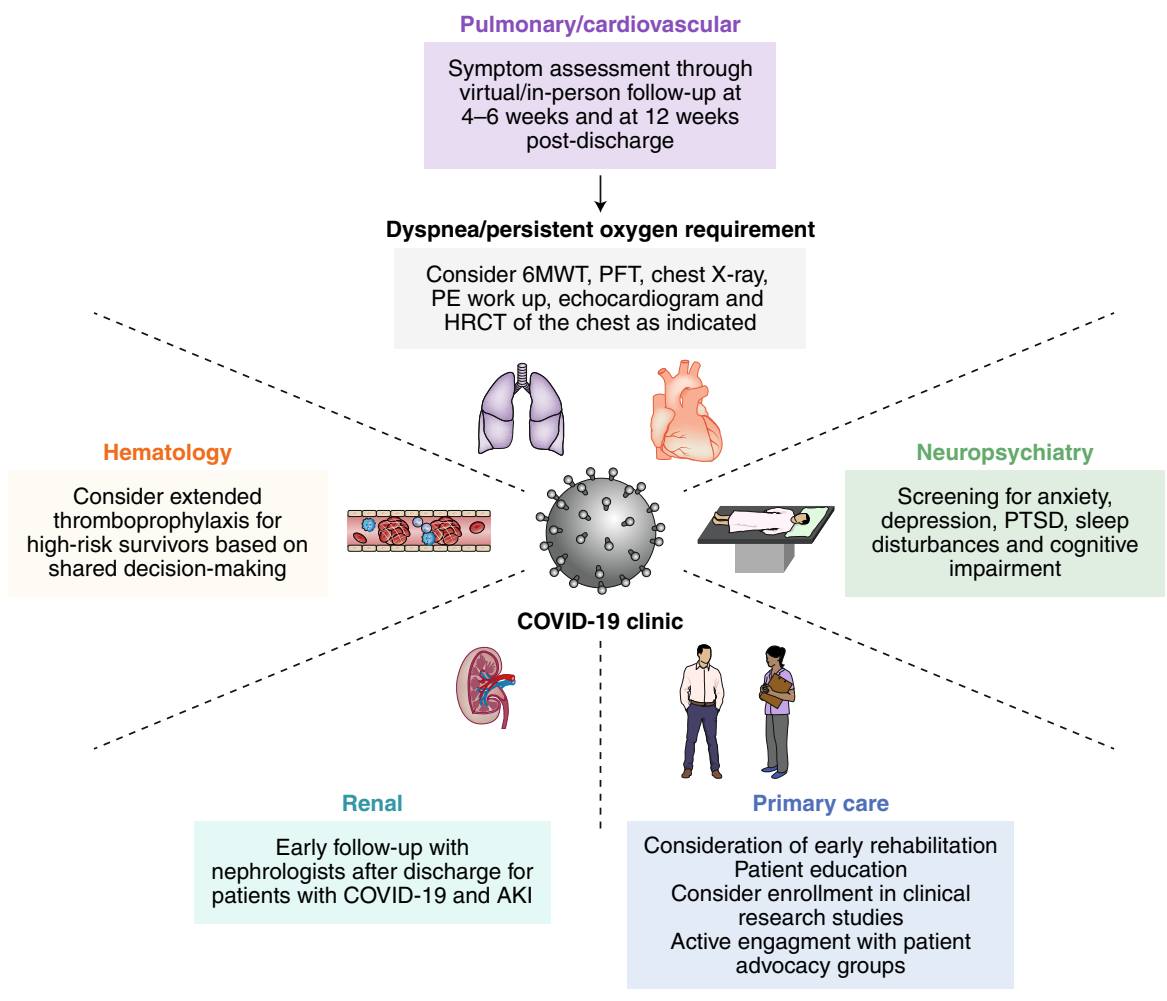

Fig. 2 | Interdisciplinary management in COVID-19 clinics. Multidisciplinary collaboration is essential to provide integrated outpatient care to survivors of acute COVID-19 in COVID-19 clinics. Depending on resources, prioritization may be considered for those at high risk for post-acute COVID-19, defined as those with severe illness during acute COVID-19 and/or requirement for care in an ICU, advanced age and the presence of organ comorbidities (pre-existing respiratory disease, obesity, diabetes, hypertension, chronic cardiovascular disease, chronic kidney disease, post-organ transplant or active cancer). The pulmonary/cardiovascular management plan was adapted from a guidance document for patients hospitalized with COVID-19 pneumonia ${ }^{76}$. $\mathrm{HRCT}$, high-resolution computed tomography; PE, pulmonary embolism.

their association with post-acute COVID-19 outcomes in those who have recovered remains to be determined.

\section{Pathophysiology}

The predominant pathophysiologic mechanisms of acute COVID19 include the following: direct viral toxicity; endothelial damage and microvascular injury; immune system dysregulation and stimulation of a hyperinflammatory state; hypercoagulability with resultant in situ thrombosis and macrothrombosis; and maladaptation of the angiotensin-converting enzyme 2 (ACE2) pathway ${ }^{2}$. The overlap of sequelae of post-acute COVID-19 with those of SARS and MERS may be explained by phylogenetic similarities between the responsible pathogenic coronaviruses. The overlap of genomic sequence identity of SARS-CoV-2 is $79 \%$ with SARS-CoV-1 and $50 \%$ with MERS-CoV ${ }^{28,29}$. Moreover, SARS-CoV-1 and SARS-CoV-2 share the same host cell receptor: ACE2. However, there are notable differences, such as the higher affinity of SARS-CoV-2 for ACE2 compared with SARS-CoV-1, which is probably due to differences in the receptor-binding domain of the spike protein that mediates contact with ACE2. In contrast with the other structural genes, the spike gene has diverged in SARS-CoV-2, with only $73 \%$ amino acid similarity with SARS-CoV-1 in the receptor-binding domain of the spike protein ${ }^{30}$. Moreover, an additional S1-S2 cleavage site in SARS-CoV-2 enables more effective cleavage by host proteases and facilitates more effective binding ${ }^{30,31}$. These mechanisms have probably contributed to the more effective and widespread transmission of SARS-CoV-2.

Potential mechanisms contributing to the pathophysiology of post-acute COVID-19 include: (1) virus-specific pathophysiologic changes; (2) immunologic aberrations and inflammatory damage in response to the acute infection; and (3) expected sequelae of post-critical illness. While the first two are discussed in more detail in the organ-specific sections below, post-intensive care syndrome is now well recognized and includes new or worsening abnormalities in physical, cognitive and psychiatric domains after critical illness ${ }^{32-36}$. The pathophysiology of post-intensive care syndrome is multifactorial and has been proposed to involve microvascular ischemia and injury, immobility and metabolic alterations during critical illness ${ }^{34}$. Additionally, similar to previous studies of SARS survivors, $25-30 \%$ of whom experienced secondary infections ${ }^{37,38}$, survivors of acute COVID-19 may be at increased risk of infections with bacterial, fungal (pulmonary aspergillosis) or other pathogens $^{39-41}$. However, these secondary infections do not explain the persistent and prolonged sequelae of post-acute COVID-19.

\section{Pulmonary sequelae}

Epidemiology and clinical manifestations. A spectrum of pulmonary manifestations, ranging from dyspnea (with or without chronic oxygen dependence) to difficult ventilator weaning and fibrotic lung damage, has been reported among COVID-19 survivors. Similar to survivors of acute respiratory distress syndrome (ARDS) from other etiologies, dyspnea is the most common persistent symptom beyond acute COVID-19, ranging from $42-66 \%$ prevalence at $60-100 \mathrm{~d}$ follow-up ${ }^{3,20,24,26}$. In the post-acute COVID19 Chinese study, the median 6-min walking distance was lower than normal reference values in approximately one-quarter of patients at 6 months ${ }^{5}-$ a prevalence similar to that in SARS and MERS survivors 9 . The need for supplemental oxygen due to persistent hypoxemia, or new requirement for continuous positive airway pressure or other breathing support while sleeping, was reported 


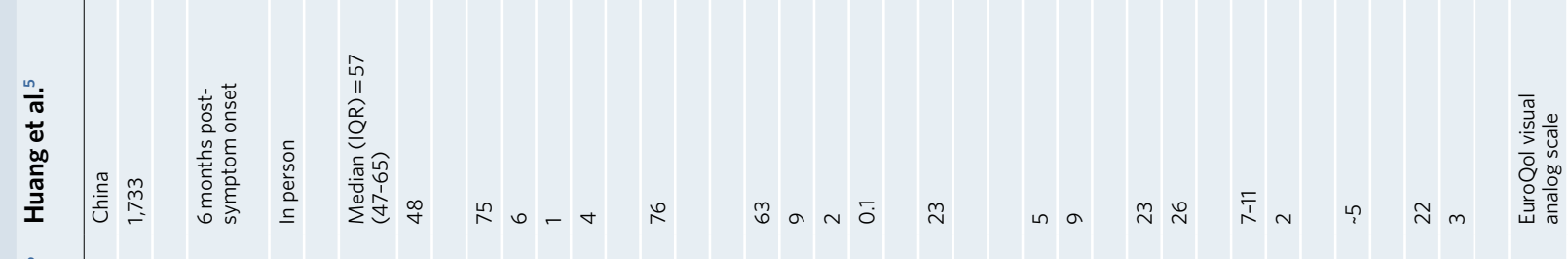

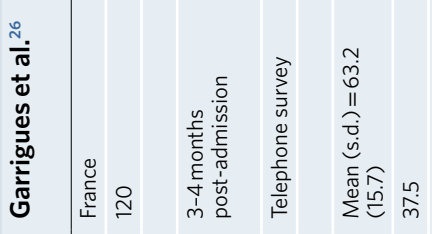

ㄴ

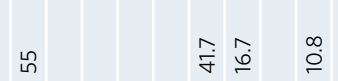

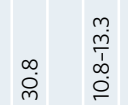

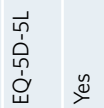

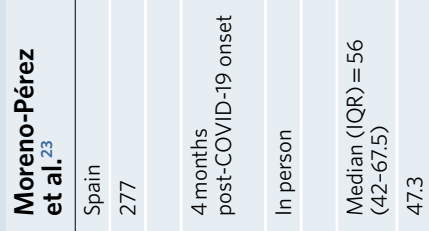

$\hat{\infty}$

$\Xi \bar{N}$

in

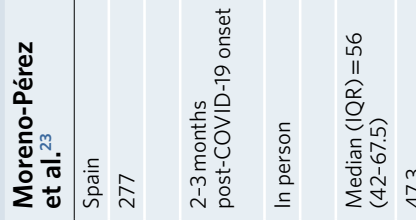

कo

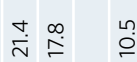

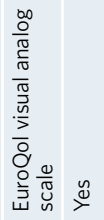

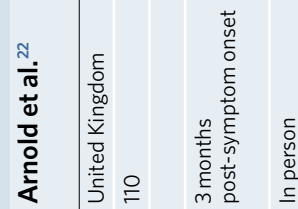

욜

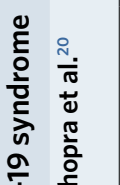

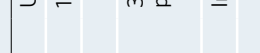

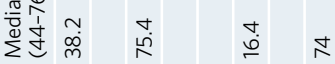

กิ $\stackrel{n}{+}$

$\stackrel{\infty}{m} \stackrel{\infty}{=} \stackrel{\grave{j}}{=}$

ন $\stackrel{\infty}{\stackrel{\infty}{ }} \stackrel{\circ}{\circ}$

密

ঠे

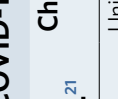

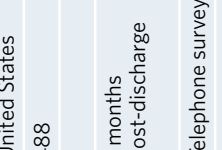

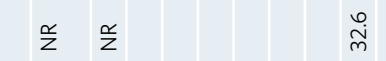

๙ิ

$\overline{\underline{m}}$

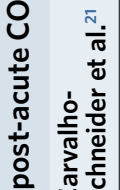

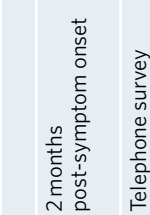

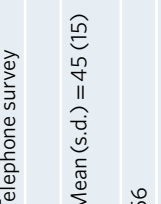

(1)

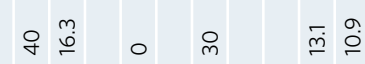

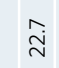

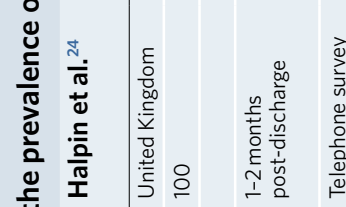

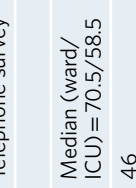

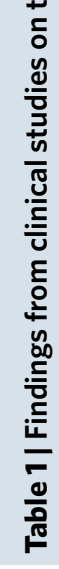

$\stackrel{\infty}{\wedge}$ -

d

우

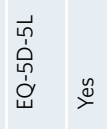

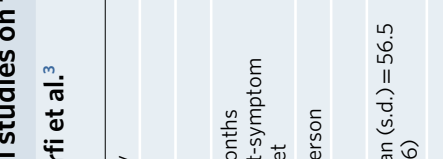

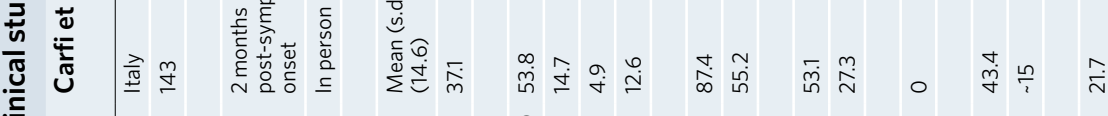

$\frac{n}{1} \div$
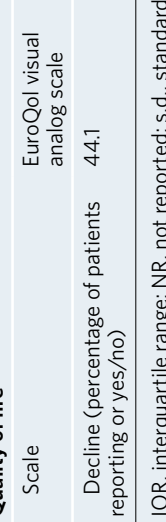
in 6.6 and $6.9 \%$ of patients, respectively, at $60 \mathrm{~d}$ follow-up in the post-acute COVID-19 US study ${ }^{20}$. Among 1,800 patients requiring tracheostomies during acute COVID-19, only 52\% were successfully weaned from mechanical ventilation 1 month later in a national cohort study from Spain ${ }^{42}$. A reduction in diffusion capacity is the most commonly reported physiologic impairment in post-acute COVID-19, with significant decrement directly related to the severity of acute illness ${ }^{5,43-46}$, which is consistent with studies of SARS and MERS survivors ${ }^{9}$, mild H1N1 influenza survivors ${ }^{47}$ and historical ARDS survivors ${ }^{48}$. Although less common, hospitalized COVID-19 survivors have been found to have restrictive pulmonary physiology at 3 and 6 months $^{5,49}$, which has also been observed in historical ARDS survivor populations ${ }^{48,50}$.

Approximately $50 \%$ of 349 patients whounderwenthigh-resolution computed tomography of the chest at 6 months had at least one abnormal pattern in the post-acute COVID-19 Chinese study ${ }^{5}$. The majority of abnormalities observed by computed tomography were ground-glass opacities. This study did not investigate chronic pulmonary embolism as computed tomography pulmonary angiograms were not obtained. The long-term risks of chronic pulmonary embolism and consequent pulmonary hypertension are unknown at this time. Fibrotic changes on computed tomography scans of the chest, consisting primarily of reticulations or traction bronchiectasis, were observed 3 months after hospital discharge in approximately 25 and $65 \%$ of survivors in cohort studies of mild-to-moderate cases ${ }^{45}$ and mostly severe cases $^{49}$, respectively, as distinguished by a requirement for supplemental oxygen. However, these prevalence estimates should be considered preliminary given the sample size of each of these cohorts. The prevalence estimates of post-acute COVID-19 sequelae from these studies suggest that patients with greater severity of acute COVID-19 (especially those requiring a high-flow nasal cannula and non-invasive or invasive mechanical ventilation) are at the highest risk for long-term pulmonary complications, including persistent diffusion impairment and radiographic pulmonary abnormalities (such as pulmonary fibrosis) ${ }^{5,22}$.

Pathology and pathophysiology. Viral-dependent mechanisms (including invasion of alveolar epithelial and endothelial cells by SARS-CoV-2) and viral-independent mechanisms (such as immunological damage, including perivascular inflammation) contribute to the breakdown of the endothelial-epithelial barrier with invasion of monocytes and neutrophils and extravasation of a protein-rich exudate into the alveolar space, consistent with other forms of ARDS ${ }^{51}$. All phases of diffuse alveolar damage have been reported in COVID-19 autopsy series, with organizing and focal fibroproliferative diffuse alveolar damage seen later in the disease course $^{52,53}$, consistent with other etiologies of ARDS ${ }^{54,55}$. Rare areas of myofibroblast proliferation, mural fibrosis and microcystic honeycombing have also been noted. This fibrotic state may be provoked by cytokines such as interleukin-6 (IL-6) and transforming growth factor- $\beta$, which have been implicated in the development of pulmonary fibrosis ${ }^{6,56-58}$ and may predispose to bacterial colonization and subsequent infection ${ }^{59-61}$. Analysis of lung tissue from five cases with severe COVID-19-associated pneumonia, including two autopsy specimens and three specimens from explanted lungs of recipients of lung transplantation, showed histopathologic and single-cell RNA expression patterns similar to end-stage pulmonary fibrosis without persistent SARS-CoV-2 infection, suggesting that some individuals develop accelerated lung fibrosis after resolution of the active infection ${ }^{62}$.

Pulmonary vascular microthrombosis and macrothrombosis have been observed in $20-30 \%$ of patients with COVID-19 (refs. ${ }^{63-67}$ ), which is higher than in other critically ill patient populations $(1-10 \%)^{68,69}$. In addition, the severity of endothelial injury and widespread thrombosis with microangiopathy seen on lung autopsy is greater than that seen in ARDS from influenza ${ }^{70,71}$.
Management considerations. Post-hospital discharge care of COVID-19 survivors has been recognized as a major research priority by professional organizations ${ }^{72}$, and guidance for the management of these patients is still evolving ${ }^{19}$. Home pulse oximetry using Food and Drug Administration-approved devices has been suggested as a useful tool for monitoring patients with persistent symptoms; however, supporting evidence is currently lacking ${ }^{73,74}$. Some experts have also proposed evaluation with serial PFTs and $6 \mathrm{MWT}$ for those with persistent dyspnea, as well as high-resolution computed tomography of the chest at 6 and 12 months $^{75}$.

In a guidance document adopted by the British Thoracic Society, algorithms for evaluating COVID-19 survivors in the first 3 months after hospital discharge are based on the severity of acute COVID-19 and whether or not the patient received ICU-level care ${ }^{76}$. Algorithms for both severe and mild-to-moderate COVID-19 groups recommend clinical assessment and chest X-ray in all patients at 12 weeks, along with consideration of PFTs, 6MWTs, sputum sampling and echocardiogram according to clinical judgment. Based on this 12 -week assessment, patients are further recommended to be evaluated with high-resolution computed tomography of the chest, computed tomography pulmonary angiogram or echocardiogram, or discharged from follow-up. In addition to this 12-week assessment, an earlier clinical assessment for respiratory, psychiatric and thromboembolic sequelae, as well as rehabilitation needs, is also recommended at 4-6weeks after discharge for those with severe acute COVID-19, defined as those who had severe pneumonia, required ICU care, are elderly or have multiple comorbidities.

Treatment with corticosteroids may be beneficial in a subset of patients with post-COVID inflammatory lung disease, as suggested by a preliminary observation of significant symptomatic and radiological improvement in a small UK cohort of COVID19 survivors with organizing pneumonia at 6 weeks after hospital discharge ${ }^{77}$. Steroid use during acute COVID-19 was not associated with diffusion impairment and radiographic abnormalities at 6 months follow-up in the post-acute COVID-19 Chinese study 5 . Lung transplantation has previously been performed for fibroproliferative lung disease after $\mathrm{ARDS}^{78}$ due to influenza A (H1N1) infection $^{79}$ and COVID-19 (refs. ${ }^{62,80}$ ). Clinical trials of antifibrotic therapies to prevent pulmonary fibrosis after COVID-19 are underway (Table 2) ${ }^{81}$.

\section{Hematologic sequelae}

Epidemiology and clinical manifestations. Retrospective data on post-acute thromboembolic events, although limited by small sample size, variability in outcome ascertainment and inadequate systematic follow-up, suggest the rate of venous thromboembolism (VTE) in the post-acute COVID-19 setting to be $<5 \%$. A single-center report of 163 patients from the United States without post-discharge thromboprophylaxis suggested a $2.5 \%$ cumulative incidence of thrombosis at $30 \mathrm{~d}$ following discharge, including segmental pulmonary embolism, intracardiac thrombus, thrombosed arteriovenous fistula and ischemic stroke ${ }^{82}$. The median duration to these events was $23 \mathrm{~d}$ post-discharge. In this same study, there was a $3.7 \%$ cumulative incidence of bleeding at $30 \mathrm{~d}$ post-discharge, mostly related to mechanical falls. Similar VTE rates have been reported in retrospective studies from the United Kingdom ${ }^{83,84}$. A prospective study from Belgium at 6 weeks post-discharge follow-up assessed D-dimer levels and venous ultrasound in 102 patients; $8 \%$ received post-discharge thromboprophylaxis ${ }^{85}$. Only one asymptomatic VTE event was reported. Similarly, no DVT was seen in 390 participants (selected using a stratified sampling procedure to include those with a higher severity of acute COVID-19) who had ultrasonography of lower extremities in the post-acute COVID-19 Chinese study 5 . Larger ongoing studies, such as CORONA-VTE, CISCO-19 and CORE-19, will help to establish more definitive rates of such complications ${ }^{86,87}$. 


\section{Table 2 | Active research studies and questions pertaining to post-acute COVID-19}

Question

\section{General}

What are the long-term sequelae of COVID-19?

What are the immunologic, enzymatic, metabolic and radiographic predictors of post-acute COVID-19?

What are the long-term effects of COVID-19 on health-related quality of life?

What are the long-term effects of COVID-19 on functional exercise capacity?

\section{Pulmonary}

Is there a role for antifibrotic therapy for the prevention of development of pulmonary fibrosis and other respiratory complications in COVID-19 survivors?

Does pulmonary rehabilitation improve pulmonary outcomes in post-acute COVID-19?

\section{Hematologic}

Does extended thromboprophylaxis lead to clinically meaningful benefit with regards to post-hospital discharge VTE in patients with COVID-19?

Does prolonged thromboprophylaxis lead to clinically meaningful benefit with regards to venous thromboembolic events in outpatients with COVID-19?

Do anti-platelets such as aspirin have a role in primary thromboprophylaxis in patients with COVID-19 managed as outpatients?

\section{Cardiovascular}

What are the medium- and long-term effects of COVID-19 on biventricular cardiac function?

\section{Neuropsychiatric}

What are the physical examination and brain-imaging characteristics in those with persistent neurological symptoms in post-acute COVID-19?

What are the long-term psychiatric sequelae of COVID-19?

\section{Renal}

What are the short- and long-term renal outcomes and their predictors in COVID-19 survivors?

\section{Gastrointestinal and hepatobiliary}

What are the long-term consequences of COVID-19 on gastrointestinal symptoms, post-infection irritable bowel syndrome and dyspepsia?

\section{Study name and/or ID}

\author{
COVIDOM (NCT04679584) \\ CO-Qo-ICU (NCT04401111) \\ MOIST (NCT04525404) \\ LIINC (NCT04362150) \\ NCT04411147 \\ NCT0 4573062 \\ NCT04605757
}

BIOMARK-COVID (NCT04664023)

MOIST (NCT04525404)

COVIDOM (NCT04679584)

RECOVER-19 (NCT04456036)

CO-Qo-ICU (NCT04401111)

COREG Extension (NCT04602260)

NCT04586413

NCT04632355

CO-Qo-ICU (NCT04401111)

COREG Extension (NCT04602260)

\section{NCT04652518}

NCT04282902

NCT04541680

NCT04527354

NCT04649918

NCT04365738

NCT04406532

NCT04642040

NCT04508439

COVID-PREVENT (NCT04416048)

ACTIV4 (NCT04498273)

PREVENT-HD (NCT04508023)

ACTIV4 (NCT04498273)

CO-Qo-ICU (NCT04401111)

MOIST (NCT04525404)

\author{
NCT04564287 \\ CO-Qo-ICU (NCTO4401111) \\ NCT04632355 \\ MIND/COVID-19 (NCT04556565)
}

\author{
NCT04353583 \\ CO-Qo-ICU (NCT04401111) \\ MOIST (NCT04525404)
}

NCT04691895

aStudy IDs are for ClinicalTrials.gov.

Pathology and pathophysiology. Unlike the consumptive coagulopathy characteristic of disseminated intravascular coagulation, COVID-19-associated coagulopathy is consistent with a hyperinflammatory and hypercoagulable state ${ }^{88,89}$. This may explain the disproportionately high rates $(20-30 \%)$ of thrombotic rather than bleeding complications in acute COVID-19 (ref. ${ }^{90}$ ). Mechanisms of thromboinflammation include endothelial injury ${ }^{70,91-93}$, complement activation ${ }^{94-96}$, platelet activation and platelet-leukocyte interactions $s^{97-99}$, neutrophil extracellular traps ${ }^{95,100,101}$, release of pro-inflammatory cytokines ${ }^{102}$, disruption of normal coagulant 
pathways $^{103}$ and hypoxia ${ }^{104}$, similar to the pathophysiology of thrombotic microangiopathy syndromes ${ }^{105}$. The risk of thrombotic complications in the post-acute COVID-19 phase is probably linked to the duration and severity of a hyperinflammatory state, although how long this persists is unknown.

Management considerations. Although conclusive evidence is not yet available, extended post-hospital discharge (up to 6 weeks) and prolonged primary thromboprophylaxis (up to $45 \mathrm{~d}$ ) in those managed as outpatients may have a more favorable risk-benefit ratio in COVID-19 given the noted increase in thrombotic complications during the acute phase, and this is an area of active investigation (NCT04508439, COVID-PREVENT (NCT04416048), ACTIV4 (NCT04498273) and PREVENT-HD (NCT04508023) $)^{106,107 .}$ Elevated D-dimer levels (greater than twice the upper limit of normal), in addition to comorbidities such as cancer and immobility, may help to risk stratify patients at the highest risk of post-acute thrombosis; however, individual patient-level considerations for risk versus benefit should dictate recommendations at this time ${ }^{86,108-110}$.

Direct oral anticoagulants and low-molecular-weight heparin are preferred anticoagulation agents over vitamin $\mathrm{K}$ antagonists due to the lack of need to frequently monitor therapeutic levels, as well as the lower risk of drug-drug interactions ${ }^{108,109}$. Therapeutic anticoagulation for those with imaging-confirmed VTE is recommended for $\geq 3$ months, similar to provoked $\mathrm{VTE}^{72,111}$. The role of antiplatelet agents such as aspirin as an alternative (or in conjunction with anticoagulation agents) for thromboprophylaxis in COVID-19 has not yet been defined and is currently being investigated as a prolonged primary thromboprophylaxis strategy in those managed as outpatients (ACTIV4 (NCT04498273)). Physical activity and ambulation should be recommended to all patients when appropriate ${ }^{102}$.

\section{Cardiovascular sequelae}

Epidemiology and clinical manifestations. Chest pain was reported in up to $\sim 20 \%$ of COVID-19 survivors at $60 \mathrm{~d}$ follow-up ${ }^{3,21}$, while ongoing palpitations and chest pain were reported in 9 and $5 \%$, respectively, at 6 months follow-up in the post-acute COVID19 Chinese study ${ }^{5}$. An increased incidence of stress cardiomyopathy has been noted during the COVID-19 pandemic compared with pre-pandemic periods ( 7.8 versus $1.5-1.8 \%$, respectively), although mortality and re-hospitalization rates in these patients are similiar ${ }^{112}$. Preliminary data with cardiac magnetic resonance imaging (MRI) suggest that ongoing myocardial inflammation may be present at rates as high as $60 \%$ more than 2 months after a diagnosis of COVID-19 at a COVID-testing center, although the reproducibility and consistency of these data have been debated ${ }^{113}$. In a study of 26 competitive college athletes with mild or asymptomatic SARS-CoV-2 infection, cardiac MRI revealed features diagnostic of myocarditis in $15 \%$ of participants, and previous myocardial injury in $30.8 \%$ of participants ${ }^{114}$.

Pathology and pathophysiology. Mechanisms perpetuating cardiovascular sequelae in post-acute COVID-19 include direct viral invasion, downregulation of ACE2, inflammation and the immunologic response affecting the structural integrity of the myocardium, pericardium and conduction system. Autopsy studies in 39 cases of COVID-19 detected virus in the heart tissue of $62.5 \%$ of patients ${ }^{115}$. The subsequent inflammatory response may lead to cardiomyocyte death and fibro-fatty displacement of desmosomal proteins important for cell-to-cell adherence ${ }^{116,117}$.

Recovered patients may have persistently increased cardiometabolic demand, as observed in long-term evaluation of SARS survivors $^{118}$. This may be associated with reduced cardiac reserve, corticosteroid use and dysregulation of the renin-angiotensinaldosterone system (RAAS). Myocardial fibrosis or scarring, and resultant cardiomyopathy from viral infection, can lead to re-entrant arrhythmias $^{119}$. COVID-19 may also perpetuate arrhythmias due to a heightened catecholaminergic state due to cytokines such as IL-6, IL- 1 and tumor necrosis factor- $\alpha$, which can prolong ventricular action potentials by modulating cardiomyocyte ion channel expression ${ }^{120}$. Autonomic dysfunction after viral illness, resulting in postural orthostatic tachycardia syndrome and inappropriate sinus tachycardia, has previously been reported as a result of adrenergic modulation ${ }^{121,122}$.

Management considerations. Serial clinical and imaging evaluation with electrocardiogram and echocardiogram at 4-12 weeks may be considered in those with cardiovascular complications during acute infection, or persistent cardiac symptoms ${ }^{76,123}$. Current evidence does not support the routine utilization of advanced cardiac imaging, and this should be considered on a case-by-case basis. Recommendations for competitive athletes with cardiovascular complications related to COVID-19 include abstinence from competitive sports or aerobic activity for 3-6months until resolution of myocardial inflammation by cardiac MRI or troponin normalization ${ }^{124,125}$.

Despite initial theoretical concerns regarding increased levels of ACE2 and the risk of acute COVID-19 with the use of RAAS inhibitors, they have been shown to be safe and should be continued in those with stable cardiovascular disease ${ }^{126,127}$. Instead, abrupt cessation of RAAS inhibitors may be potentially harmful ${ }^{128}$. In patients with ventricular dysfunction, guideline-directed medical therapy should be initiated and optimized as tolerated ${ }^{129}$. Withdrawal of guideline-directed medical therapy was associated with higher mortality in the acute to post-acute phase in a retrospective study of 3,080 patients with COVID-19 (ref. ${ }^{130}$ ). Patients with postural orthostatic tachycardia syndrome and inappropriate sinus tachycardia may benefit from a low-dose beta blocker for heart rate management and reducing adrenergic activity ${ }^{131}$. Attention is warranted to the use of drugs such as anti-arrhythmic agents (for example, amiodarone) in patients with fibrotic pulmonary changes after COVID-19 (ref. ${ }^{132}$ ).

\section{Neuropsychiatric sequelae}

Epidemiology and clinical manifestations. Similar to chronic post-SARS syndrome, COVID-19 survivors have reported a post-viral syndrome of chronic malaise, diffuse myalgia, depressive symptoms and non-restorative sleep ${ }^{133,134}$. Other post-acute manifestations of COVID-19 include migraine-like headaches ${ }^{135,136}$ (often refractory to traditional analgesics ${ }^{137}$ ) and late-onset headaches ascribed to high cytokine levels. In a follow-up study of 100 patients, approximately $38 \%$ had ongoing headaches after 6 weeks ${ }^{138}$. Loss of taste and smell may also persist after resolution of other symptoms in approximately one-tenth of patients at up to 6 months follow-up $5,20,22,26$. Cognitive impairment has been noted with or without fluctuations, including brain fog, which may manifest as difficulties with concentration, memory, receptive language and/or executive function ${ }^{139-141}$.

Individuals with COVID-19 experience a range of psychiatric symptoms persisting or presenting months after initial infection ${ }^{142}$. In a cohort of 402 COVID-19 survivors in Italy 1 month after hospitalization, approximately $56 \%$ screened positive in at least one of the domains evaluated for psychiatric sequelae (PTSD, depression, anxiety, insomnia and obsessive compulsive symptomatology $)^{143}$. Clinically significant depression and anxiety were reported in approximately $30-40 \%$ of patients following COVID-19, similar to patients with previous severe coronavirus infections s $^{11,12,15,143,144}$. Anxiety, depression and sleep difficulties were present in approximately one-quarter of patients at 6 months follow-up in the post-acute COVID-19 Chinese study ${ }^{5}$. Notably, clinically significant PTSD symptoms were reported in approximately $30 \%$ of patients with COVID-19 requiring hospitalization, and may present early during acute infection or months later ${ }^{143,144}$. A real-world, large-scale 
dataset analysis of 62,354 COVID-19 survivors from 54 healthcare organizations in the United States estimated the incidence of first and recurrent psychiatric illness between 14 and $90 \mathrm{~d}$ of diagnosis to be $18.1 \%^{145}$. More importantly, it reported the estimated overall probability of diagnosis of a new psychiatric illness within $90 \mathrm{~d}$ after COVID-19 diagnosis to be $5.8 \%$ (anxiety disorder $=4.7 \%$; $\operatorname{mood}$ disorder $=2 \%$; insomnia $=1.9 \%$; dementia (among those $\geq 65$ years old) $=1.6 \%$ ) among a subset of 44,759 patients with no known previous psychiatric illness. These values were all significantly higher than in matched control cohorts of patients diagnosed with influenza and other respiratory tract infections.

Similar to other critical illnesses, the complications of acute COVID-19, such as ischemic or hemorrhagic stroke ${ }^{146}$, hypoxicanoxic damage, posterior reversible encephalopathy syndrome ${ }^{147}$ and acute disseminated myelitis ${ }^{148,149}$, may lead to lingering or permanent neurological deficits requiring extensive rehabilitation. Additionally, acute critical illness myopathy and neuropathies resulting during acute COVID-19 or from the effect of neuromuscular blocking agents can leave residual symptoms persisting for weeks to months ${ }^{36,150}$.

Pathology and pathophysiology. The mechanisms contributing to neuropathology in COVID-19 can be grouped into overlapping categories of direct viral infection, severe systemic inflammation, neuroinflammation, microvascular thrombosis and neurodegeneration ${ }^{139,151-153}$. While viral particles in the brain have previously been reported with other coronavirus infections ${ }^{154}$, there is not yet compelling evidence of SARS-CoV-2 infecting neurons. However, autopsy series have shown that SARS-CoV-2 may cause changes in brain parenchyma and vessels, possibly by effects on bloodbrain and blood-cerebrospinal fluid barriers, which drive inflammation in neurons, supportive cells and brain vasculature ${ }^{155,156}$. Furthermore, levels of immune activation directly correlate with cognitive-behavioral changes ${ }^{157}$. Inflammaging (a chronic low-level brain inflammation), along with the reduced ability to respond to new antigens and an accumulation of memory $\mathrm{T}$ cells (hallmarks of immunosenescence in aging and tissue injury ${ }^{158}$ ), may play a role in persistent effects of COVID-19. Other proposed mechanisms include dysfunctional lymphatic drainage from circumventricular organs $s^{159}$, as well as viral invasion in the extracellular spaces of olfactory epithelium and passive diffusion and axonal transport through the olfactory complex ${ }^{160}$. Biomarkers of cerebral injury, such as elevated peripheral blood levels of neurofilament light chain, have been found in patients with COVID-19 (ref. ${ }^{161}$ ), with a more sustained increase in severe infections ${ }^{162}$, suggesting the possibility of more chronic neuronal injury.

Post-COVID brain fog in critically ill patients with COVID19 may evolve from mechanisms such as deconditioning or PTSD $^{141}$. However, reports of COVID-19 brain fog after mild COVID-19 suggest that dysautonomia may contribute as well ${ }^{163,164}$. Finally, long-term cognitive impairment is well recognized in the post-critical illness setting, occurring in $20-40 \%$ of patients discharged from an $\mathrm{ICU}^{165}$.

Management considerations. Standard therapies should be implemented for neurologic complications such as headaches, with imaging evaluation and referral to a specialist reserved for refractory headach $\mathrm{e}^{166}$. Further neuropsychological evaluation should be considered in the post-acute illness setting in patients with cognitive impairment. Standard screening tools should be used to identify patients with anxiety, depression, sleep disturbances, PTSD, dysautonomia and fatigue $\mathrm{f}^{76,141}$.

\section{Renal sequelae}

Epidemiology and clinical manifestations. Severe acute kidney injury (AKI) requiring renal replacement therapy (RRT) occurs in
$5 \%$ of all hospitalized patients and $20-31 \%$ of critically ill patients with acute COVID-19, particularly among those with severe infections requiring mechanical ventilation ${ }^{167-170}$. Early studies with short-term follow-up in patients requiring RRT showed that $27-64 \%$ were dialysis independent by $28 \mathrm{~d}$ or ICU discharge ${ }^{169,171}$. Decreased estimated glomerular filtration rate (eGFR; defined as $<90 \mathrm{ml} \mathrm{min}^{-1}$ per $1.73 \mathrm{~m}^{2}$ ) was reported in $35 \%$ of patients at 6 months in the post-acute COVID-19 Chinese study, and 13\% developed new-onset reduction of eGFR after documented normal renal function during acute COVID-19 (ref. ${ }^{5}$ ). With adequate longer-term follow-up data, those patients who require RRT for severe AKI experience high mortality, with a survival probability of 0.46 at $60 \mathrm{~d}$ and rates of renal recovery reportedly at $84 \%$ among survivors ${ }^{170}$.

Pathology and pathophysiology. SARS-CoV-2 has been isolated from renal tissue ${ }^{172}$, and acute tubular necrosis is the primary finding noted from renal biopsies ${ }^{173,174}$ and autopsies ${ }^{175,176}$ in COVID-19. COVID-19-associated nephropathy (COVAN) is characterized by the collapsing variant of focal segmental glomerulosclerosis, with involution of the glomerular tuft in addition to acute tubular injury, and is thought to develop in response to interferon and chemokine activation $^{177,178}$. Association with APOL1 risk alleles suggests that SARS-CoV-2 acts as a second hit in susceptible patients, in a manner similar to human immunodeficiency virus and other viruses ${ }^{177}$. Thrombi in the renal microcirculation may also potentially contribute to the development of renal injury ${ }^{179}$.

Management considerations. While the burden of dialysisdependent AKI at the time of discharge is low, the extent of the recovery of renal function remains to be seen. As a result, COVID-19 survivors with persistent impaired renal function in the post-acute infectious phase may benefit from early and close follow-up with a nephrologist in AKI survivor clinics, supported by its previous association with improved outcomes ${ }^{180,181}$.

\section{Endocrine sequelae}

Epidemiology and clinical manifestations. Diabetic ketoacidosis (DKA) has been observed in patients without known diabetes mellitus weeks to months after resolution of COVID-19 symptoms ${ }^{182}$. It is not yet known how long the increased severity of pre-existing diabetes or predisposition to DKA persists after infection, and this will be addressed by the international CoviDiab registry ${ }^{183}$. Similarly, subacute thyroiditis with clinical thyrotoxicosis has been reported weeks after the resolution of respiratory symptoms ${ }^{184,185}$. COVID19 may also potentiate latent thyroid autoimmunity manifesting as new-onset Hashimoto’s thyroiditis ${ }^{186}$ or Graves' disease ${ }^{187}$.

Pathology and pathophysiology. Endocrine manifestations in the post-acute COVID-19 setting may be consequences of direct viral injury, immunological and inflammatory damage, as well as iatrogenic complications. Pre-existing diabetes may first become apparent during the acute phase of COVID-19 and can generally be treated long term with agents other than insulin, even if initially associated with DKA. There is no concrete evidence of lasting damage to pancreatic $\beta$ cells $^{188}$. Although some surveys have shown ACE2 and transmembrane serine protease (TMPRSS2; the protease involved in SARS-CoV-2 cell entry) expression in $\beta$ cells ${ }^{189}$, the primary deficit in insulin production is probably mediated by factors such as inflammation or the infection stress response, along with peripheral insulin resistance ${ }^{188}$. So far, there is no evidence that COVID-19-associated diabetes can be reversed after the acute phase, nor that its outcomes differ in COVID-19 long haulers. COVID-19 also presents risk factors for bone demineralization related to systemic inflammation, immobilization, exposure to corticosteroids, vitamin D insufficiency and interruption of antiresorptive or anabolic agents for osteoporosis ${ }^{190}$. 
Management considerations. Serologic testing for type 1 diabetesassociated autoantibodies and repeat post-prandial C-peptide measurements should be obtained at follow-up in patients with newly diagnosed diabetes mellitus in the absence of traditional risk factors for type 2 diabetes, whereas it is reasonable to treat patients with such risk factors akin to ketosis-prone type 2 diabetes ${ }^{191}$. Incident hyperthyroidism due to SARS-CoV-2-related destructive thyroiditis can be treated with corticosteroids but new-onset Graves' disease should also be ruled out ${ }^{184}$.

\section{Gastrointestinal and hepatobiliary sequelae}

Significant gastrointestinal and hepatobiliary sequelae have not been reported in COVID-19 survivors $^{22}$. Prolonged viral fecal shedding occurs in COVID-19, with viral ribonucleic acid detectable for a mean duration of $28 \mathrm{~d}$ after the onset of SARS-CoV-2 infection symptoms and persisting for a mean of $11 \mathrm{~d}$ after negative respiratory samples ${ }^{192-195}$.

COVID-19 has the potential to alter the gut microbiome, including enrichment of opportunistic infectious organisms and depletion of beneficial commensals ${ }^{196,197}$. The ability of the gut microbiota to alter the course of respiratory infections (gut-lung axis) has been recognized previously in influenza and other respiratory infections ${ }^{198}$. In COVID-19, Faecalibacterium prausnitzii, a butyrate-producing anaerobe typically associated with good health, has been inversely correlated with disease severity ${ }^{196,199}$. Studies are currently evaluating the long-term consequences of COVID-19 on the gastrointestinal system, including post-infectious irritable bowel syndrome and dyspepsia (NCT04691895).

\section{Dermatologic sequelae}

Dermatologic manifestations of COVID-19 occurred after (64\%) or concurrent to (15\%) other acute COVID-19 symptoms in an international study of 716 patients with COVID-19 (ref. ${ }^{200}$ ), with an average latency from the time of upper respiratory symptoms to dermatologic findings of $7.9 \mathrm{~d}$ in adults ${ }^{201}$. Only $3 \%$ of patients noted a skin rash at 6 months follow-up in the post-acute COVID19 Chinese study $^{5}$. The predominant dermatologic complaint was hair loss, which was noted in approximately $20 \%$ of patients ${ }^{5,26}$. Hair loss can possibly be attributed to telogen effluvium resulting from viral infection or a resultant stress response ${ }^{5}$. Ongoing investigations may provide insight into potential immune or inflammatory mechanisms of disease ${ }^{202}$.

\section{Multisystem inflammatory syndrome in children (MIS-C)} Epidemiology and clinical manifestations. MIS-C, also referred to as pediatric inflammatory multisystem syndrome temporally associated with SARS-CoV-2 (PIMS-TS), is defined by the presence of the following symptoms in people $<21$ years old (or $\leq 19$ years old per the World Health Organization definition): fever; elevated inflammatory markers; multiple organ dysfunction; current or recent SARS-CoV-2 infection; and exclusion of other plausible diagnoses $^{203,204}$. Clinical presentations of MIS-C include fever, abdominal pain, vomiting, diarrhea, skin rash, mucocutaneous lesions, hypotension and cardiovascular and neurologic compromise ${ }^{205,206}$. Overlapping features have been noted with Kawasaki disease, an acute pediatric medium-vessel vasculitis ${ }^{207}$. However, comparison of Kawasaki disease and MIS-C cohorts demonstrates distinctive epidemiologic and clinical characteristics. While $80 \%$ of Kawasaki disease cases occur in children $<5$ years of age and primarily of Asian descent ${ }^{207}$, patients with MIS-C are typically $>7$ years, encompass a broader age range and are of African, Afro-Caribbean or Hispanic origin ${ }^{206,208}$. A comparable incidence of coronary artery aneurysm and dilation has been noted among MIS-C and Kawasaki disease (20 and $25 \%$, respectively $)^{206}$. Neurological complications of MIS-C, such as headache, altered mental status, encephalopathy, cranial nerve palsies, stroke, seizure, reduced reflexes, and muscle weakness, appear to be more frequent than in Kawasaki disease ${ }^{209,210}$. A pooled meta-analysis of MIS-C studies reported recovery in $91.1 \%$ and death in $3.5 \%$ of patients ${ }^{205}$. Ongoing studies are evaluating long-term sequelae in these children (NCT04330261).

Pathology and pathophysiology. The timing of the emergence of MIS-C (which was lagging approximately 1 month behind peak COVID-19 incidence in epicenters in Spring 2020211) and the finding that most patients are negative for acute infection but are antibody positive suggest that MIS-C may result from an aberrant acquired immune response rather than acute viral infection ${ }^{208}$. Insights into the pathophysiology of MIS-C may be derived in part from Kawasaki disease and toxic shock syndrome, with possible mechanisms of injury related to immune complexes, complement activation, autoantibody formation through viral host mimicry, and massive cytokine release related to superantigen stimulation of T cells ${ }^{205,211}$.

Management considerations. Current recommendations include immunomodulatory therapy with intravenous immunoglobulin, adjunctive glucocorticoids and low-dose aspirin until coronary arteries are confirmed normal at least 4 weeks after diagnosis ${ }^{206}$. Therapeutic anticoagulation with enoxaparin or warfarin and low-dose aspirin is recommended in those with a coronary artery $z$ score $\geq 10$, documented thrombosis or an ejection fraction $<35 \%$. Studies such as the Best Available Treatment Study for Inflammatory Conditions Associated with COVID-19 (ISRCTN69546370) are evaluating the optimal choice of immunomodulatory agents for treatment.

Serial echocardiographic assessment is recommended at intervals of 1-2 and 4-6 weeks after presentation ${ }^{212}$. Cardiac MRI may be indicated 2-6 months after diagnosis in those presenting with significant transient left ventricular dysfunction (ejection fraction $<50 \%$ ) in the acute phase or persistent dysfunction to assess for fibrosis and inflammation. Serial electrocardiograms and consideration of an ambulatory cardiac monitor are recommended at follow-up visits in patients with conduction abnormalities at diagnosis.

\section{Special considerations}

Racial and ethnic considerations. Acute COVID-19 has been recognized to disproportionately affect communities of $\operatorname{color}^{27,213-216}$. A total of $51.6 \%$ of survivors in the post-acute COVID-19 US study were $\mathrm{Black}^{20}$, while the BAME group comprised 19-20.9\% in the UK studies $^{22,24}$. Only one study from the United Kingdom evaluated the association of race/ethnicity and reported that individuals belonging to the BAME group were more likely to experience dyspnea than White individuals (42.1 versus $25 \%$, respectively) at 4-8 weeks post-discharge $^{24}$. Rates of PTSD were similar in BAME and White participants in this study. Emerging data also suggest that COVAN may be the predominant pattern of renal injury in individuals of African descent ${ }^{177}$. MIS-C is also known to disproportionately affect children and adolescents of African, Afro-Caribbean or Hispanic ethnicity ${ }^{206,208}$. Larger studies are required to ascertain the association between sequelae of post-acute COVID-19 and race and ethnicity.

These important differences noted in preliminary studies may be related to multiple factors, including (but not limited to) socioeconomic determinants and racial/ethnic disparities, plausible differences in the expression of factors involved in SARS-CoV-2 pathogenesis, and comorbidities. Higher nasal epithelial expression of TMPRSS2 has been reported in Black individuals compared with other self-reported races/ethnicities ${ }^{217}$. However, caution is warranted that ongoing and future studies integrate and analyze information along multiple axes (for example, clinical and socioeconomic axes, resource deficits and external stressors) to prevent inaccurate contextualization $^{218}$. The National Institute on Minority Health and Health Disparities at the National Institutes of Health has identified 
investigation of short- and long-term effects of COVID-19 on health, and how differential outcomes can be reduced among racial and ethnic groups, as a research priority ${ }^{216}$.

Nutrition and rehabilitation considerations. Severe COVID-19, similar to other critical illnesses, causes catabolic muscle wasting, feeding difficulties and frailty, each of which is associated with an increased likelihood of poor outcome ${ }^{36}$. Malnutrition has been noted in $26-45 \%$ of patients with COVID-19, as evaluated by the Malnutrition Universal Screening Tool in an Italian study ${ }^{219}$ Protocols to provide nutritional support for patients (many of whom suffered from respiratory distress, nausea, diarrhea and anorexia, with resultant reduction in food intake) continue to be refined ${ }^{220}$.

All post-acute COVID-19 follow-up studies that incorporated assessments of health-related quality of life and functional capacity measures have universally reported significant deficits in these domains, including at 6 months in the post-acute COVID-19 Chinese study ${ }^{3,5,20}$. Given the severity of the systemic inflammatory response associated with severe COVID-19 and resultant frailty, early rehabilitation programs are being evaluated in ongoing clinical studies (Table 2). They have previously been validated to be both safe and effective in critically ill patients with $\mathrm{ARDS}^{221-223}$ and in preliminary studies in COVID-19 (ref. ${ }^{224}$ ). Model COVID-19 rehabilitation units such as those in Italy are already routinely assessing acute COVID-19 survivors for swallowing function, nutritional status and measures of functional independence ${ }^{219}$.

Patient advocacy groups. Unique to this pandemic is the creation and role of patient advocacy groups in identifying persistent symptoms and influencing research and clinical attention. Such groups include COVID Advocacy Exchange (https://www.covidadvo cacyexchange.com), the National Patient Advocate Foundation COVID Care Resource Center (https://www.patientadvocate.org/ covidcare), long-haul COVID fighters Facebook groups, the Body Politic COVID-19 Support Group (https://www.wearebodypolitic. com/covid19), Survivor Corps (https://www.survivorcorps.com/) and Patient-Led Research for COVID-19 (patientresearchcovid19. com). Surveys conducted by these groups have helped to identify persistent symptoms such as brain fog, fatigue and body aches as important components of post-acute COVID-19. Additionally, they have been instrumental in highlighting the persistence of symptoms in patients with mild-to-moderate disease who did not require hospitalization ${ }^{225}$. Active engagement with these patient advocacy groups, many of whom identify themselves as long haulers, is cru$\mathrm{cial}^{226}$. Dissemination of contact information and resources of these groups can occur at pharmacies, physician offices and in discharge summaries upon hospital discharge.

\section{Conclusions and future directions}

The multi-organ sequelae of COVID-19 beyond the acute phase of infection are increasingly being appreciated as data and clinical experience in this timeframe accrue. Necessary active and future research include the identification and characterization of key clinical, serological, imaging and epidemiologic features of COVID-19 in the acute, subacute and chronic phases of disease, which will help us to better understand the natural history and pathophysiology of this new disease entity (Table 2). Active and future clinical studies, including prospective cohorts and clinical trials, along with frequent review of emerging evidence by working groups and task forces, are paramount to developing a robust knowledge database and informing clinical practice in this area. Currently, healthcare professionals caring for survivors of acute COVID-19 have the key role of recognizing, carefully documenting, investigating and managing ongoing or new symptoms, as well as following up organ-specific complications that developed during acute illness. It is also imperative that clinicians provide information in accessible formats, including clinical studies available for participation and additional resources such as patient advocacy and support groups.

Moreover, it is clear that care for patients with COVID-19 does not conclude at the time of hospital discharge, and interdisciplinary cooperation is needed for comprehensive care of these patients in the outpatient setting. As such, it is crucial for healthcare systems and hospitals to recognize the need to establish dedicated COVID19 clinics $^{74}$, where specialists from multiple disciplines are able to provide integrated care. Prioritization of follow-up care may be considered for those at high risk for post-acute COVID-19, including those who had severe illness during acute COVID-19 and/or required care in an ICU, those most susceptible to complications (for example, the elderly, those with multiple organ comorbidities, those post-transplant and those with an active cancer history) and those with the highest burden of persistent symptoms.

Given the global scale of this pandemic, it is apparent that the healthcare needs for patients with sequelae of COVID-19 will continue to increase for the foreseeable future. Rising to this challenge will require harnessing of existing outpatient infrastructure, the development of scalable healthcare models and integration across disciplines for improved mental and physical health of survivors of COVID-19 in the long term.

Received: 18 October 2020; Accepted: 9 February 2021; Published online: 22 March 2021

\section{References}

1. Dong, E., Du, H. \& Gardner, L. An interactive web-based dashboard to track COVID-19 in real time. Lancet Infect. Dis. 20, 533-534 (2020).

2. Gupta, A. et al. Extrapulmonary manifestations of COVID-19. Nat. Med. 26, 1017-1032 (2020).

3. Carfi, A., Bernabei, R., Landi, F. \& Gemelli Against COVID-19 Post-Acute Care Study Group. Persistent symptoms in patients after acute COVID-19. J. Am. Med. Assoc. 324, 603-605 (2020).

4. Tenforde, M. W. et al. Symptom duration and risk factors for delayed return to usual health among outpatients with COVID-19 in a multistate health care systems network-United States, March-June 2020. Morb. Mortal. Wkly Rep. 69, 993-998 (2020).

5. Huang, C. et al. 6-month consequences of COVID-19 in patients discharged from hospital: a cohort study. Lancet 397, 220-232 (2021).

6. McElvaney, O. J. et al. Characterization of the inflammatory response to severe COVID-19 Illness. Am. J. Respir. Crit. Care Med. 202, 812-821 (2020).

7. Sungnak, W. et al. SARS-CoV-2 entry factors are highly expressed in nasal epithelial cells together with innate immune genes. Nat. Med. 26, 681-687 (2020).

8. Tang, N., Li, D., Wang, X. \& Sun, Z. Abnormal coagulation parameters are associated with poor prognosis in patients with novel coronavirus pneumonia. J. Thromb. Haemost. 18, 844-847 (2020).

9. Ahmed, H. et al. Long-term clinical outcomes in survivors of severe acute respiratory syndrome and Middle East respiratory syndrome coronavirus outbreaks after hospitalisation or ICU admission: a systematic review and meta-analysis. J. Rehabil. Med. 52, jrm00063 (2020).

10. Hui, D. S. et al. Impact of severe acute respiratory syndrome (SARS) on pulmonary function, functional capacity and quality of life in a cohort of survivors. Thorax 60, 401-409 (2005).

11. Lam, M. H. et al. Mental morbidities and chronic fatigue in severe acute respiratory syndrome survivors: long-term follow-up. Arch. Intern. Med. 169, 2142-2147 (2009)

12. Lee, S. H. et al. Depression as a mediator of chronic fatigue and post-traumatic stress symptoms in Middle East respiratory syndrome survivors. Psychiatry Investig. 16, 59-64 (2019).

13. Moldofsky, H. \& Patcai, J. Chronic widespread musculoskeletal pain, fatigue, depression and disordered sleep in chronic post-SARS syndrome; a case-controlled study. BMC Neurol. 11, 37 (2011).

14. Ong, K.-C. et al. Pulmonary function and exercise capacity in survivors of severe acute respiratory syndrome. Eur. Respir. J. 24, 436-442 (2004).

15. Lee, A. M. et al. Stress and psychological distress among SARS survivors 1 year after the outbreak. Can. J. Psychiatry 52, 233-240 (2007).

16. Datta, S. D., Talwar, A. \& Lee, J. T. A proposed framework and timeline of the spectrum of disease due to SARS-CoV-2 infection: illness beyond acute infection and public health implications. J. Am. Med. Assoc. 324, 2251-2252 (2020). 
17. Greenhalgh, T., Knight, M., A'Court, C., Buxton, M. \& Husain, L. Management of post-acute COVID-19 in primary care. Brit. Med. J. 370, m3026 (2020).

18. Van Kampen, J. J. A. et al. Duration and key determinants of infectious virus shedding in hospitalized patients with coronavirus disease-2019 (COVID-19). Nat. Commun. 12, 267 (2021).

19. Shah, W., Hillman, T., Playford, E. D. \& Hishmeh, L. Managing the long term effects of COVID-19: summary of NICE, SIGN, and RCGP rapid guideline. Brit. Med. J. 372, n136 (2021).

20. Chopra, V., Flanders, S. A. \& O’Malley, M. Sixty-day outcomes among patients hospitalized with COVID-19. Ann. Intern. Med. https://doi.org/ 10.7326/M20-5661 (2020).

21. Carvalho-Schneider, C. et al. Follow-up of adults with noncritical COVID-19 two months after symptom onset. Clin. Microbiol. Infect. 27, 258-263 (2021).

22. Arnold, D. T. et al. Patient outcomes after hospitalisation with COVID-19 and implications for follow-up: results from a prospective UK cohort. Thorax https://doi.org/10.1136/thoraxjnl-2020-216086 (2020).

23. Moreno-Pérez, O. et al. Post-acute COVID-19 syndrome. Incidence and risk factors: a Mediterranean cohort study. J. Infect. https://doi.org/ 10.1016/j.jinf.2021.01.004 (2021).

24. Halpin, S. J. et al. Postdischarge symptoms and rehabilitation needs in survivors of COVID-19 infection: a cross-sectional evaluation. J. Med. Virol. 93, 1013-1022 (2021).

25. Jacobs, L. G. et al. Persistence of symptoms and quality of life at 35 days after hospitalization for COVID-19 infection. PLOS ONE 15, e0243882 (2020).

26. Garrigues, E. et al. Post-discharge persistent symptoms and health-related quality of life after hospitalization for COVID-19. J. Infect. 81, e4-e6 (2020).

27. Williamson, E. J. et al. Factors associated with COVID-19-related death using OpenSAFELY. Nature 584, 430-436 (2020).

28. Lu, R. et al. Genomic characterisation and epidemiology of 2019 novel coronavirus: implications for virus origins and receptor binding. Lancet 395, 565-574 (2020).

29. Hu, B., Guo, H., Zhou, P. \& Shi, Z.-L.Characteristics of SARS-CoV-2 and COVID-19. Nat. Rev. Microbiol. 19, 141-154 (2021).

30. Shang, J. et al. Structural basis of receptor recognition by SARS-CoV-2. Nature 581, 221-224 (2020).

31. Wrobel, A. G. et al. SARS-CoV-2 and bat RaTG13 spike glycoprotein structures inform on virus evolution and furin-cleavage effects. Nat. Struct. Mol. Biol. 27, 763-767 (2020).

32. Needham, D. M. et al. Physical and cognitive performance of patients with acute lung injury 1 year after initial trophic versus full enteral feeding. EDEN trial follow-up. Am. J. Respir. Crit. Care Med. 188, 567-576 (2013).

33. Pandharipande, P. P. et al. Long-term cognitive impairment after critical illness. N. Engl. J. Med. 369, 1306-1316 (2013).

34. Inoue, $\mathrm{S}$. et al. Post-intensive care syndrome: its pathophysiology, prevention, and future directions. Acute Med. Surg. 6, 233-246 (2019).

35. Kress, J. P. \& Hall, J. B. ICU-acquired weakness and recovery from critical illness. N. Engl. J. Med. 370, 1626-1635 (2014).

36. Hosey, M. M. \& Needham, D. M. Survivorship after COVID-19 ICU stay. Nat. Rev. Dis. Prim. 6, 60 (2020).

37. Zahariadis, G. et al. Risk of ruling out severe acute respiratory syndrome by ruling in another diagnosis: variable incidence of atypical bacteria coinfection based on diagnostic assays. Can. Respir. J. 13, 17-22 (2006).

38. Zheng, Z., Chen, R. \& Li, Y. The clinical characteristics of secondary infections of lower respiratory tract in severe acute respiratory syndrome. Chin. J. Respir. Crit. Care Med. 2, 270-274 (2003).

39. Huang, C. et al. Clinical features of patients infected with 2019 novel coronavirus in Wuhan, China. Lancet 395, 497-506 (2020).

40. Lescure, F. X. et al. Clinical and virological data of the first cases of COVID-19 in Europe: a case series. Lancet Infect. Dis. 20, 697-706 (2020)

41. Zhou, F. et al. Clinical course and risk factors for mortality of adult inpatients with COVID-19 in Wuhan, China: a retrospective cohort study. Lancet 395, 1054-1062 (2020).

42. Martin-Villares, C., Perez Molina-Ramirez, C., Bartolome-Benito, M., Bernal-Sprekelsen, M. \& COVID ORL ESP Collaborative Group. Outcome of 1890 tracheostomies for critical COVID-19 patients: a national cohort study in Spain. Eur. Arch. Oto Rhino Laryngol. https://doi.org/10.1007/ s00405-020-06220-3 (2020)

43. Huang, Y. et al. Impact of coronavirus disease 2019 on pulmonary function in early convalescence phase. Respir. Res. 21, 163 (2020)

44. Mo, X. et al. Abnormal pulmonary function in COVID-19 patients at time of hospital discharge. Eur. Respir. J. 55, 2001217 (2020).

45. Zhao, Y. M. et al. Follow-up study of the pulmonary function and related physiological characteristics of COVID-19 survivors three months after recovery. EClinicalMedicine 25, 100463 (2020).

46. Méndez, R. et al. Reduced diffusion capacity in COVID-19 survivors. Ann. Am. Thorac. Soc. https://doi.org/10.1513/AnnalsATS.202011-1452RL (2021).
47. Liu, W., Peng, L., Liu, H. \& Hua, S. Pulmonary function and clinical manifestations of patients infected with mild influenza A virus subtype H1N1: a one-year follow-up. PLoS ONE 10, e0133698 (2015).

48. Herridge, M. S. et al. Functional disability 5 years after acute respiratory distress syndrome. N. Engl. J. Med. 364, 1293-1304 (2011).

49. Shah, A. S. et al. A prospective study of 12-week respiratory outcomes in COVID-19-related hospitalisations. Thorax https://doi.org/10.1136/ thoraxjnl-2020-216308 (2020).

50. Burnham, E. L. et al. Chest CT features are associated with poorer quality of life in acute lung injury survivors. Crit. Care Med. 41, 445-456 (2013).

51. Huppert, L. A., Matthay, M. A. \& Ware, L. B. Pathogenesis of acute respiratory distress syndrome. Semin. Respir. Crit. Care Med. 40, 31-39 (2019).

52. Carsana, L. et al. Pulmonary post-mortem findings in a series of COVID-19 cases from northern Italy: a two-centre descriptive study. Lancet Infect. Dis. 20, 1135-1140 (2020)

53. Schaller, T. et al. Postmortem examination of patients with COVID-19. J. Am. Med. Assoc. 323, 2518-2520 (2020).

54. Burnham, E. L., Janssen, W. J., Riches, D. W., Moss, M. \& Downey, G. P. The fibroproliferative response in acute respiratory distress syndrome: mechanisms and clinical significance. Eur. Respir. J. 43, 276-285 (2014).

55. De Michele, S. et al. Forty postmortem examinations in COVID-19 patients. Am. J. Clin. Pathol. 154, 748-760 (2020).

56. Coker, R. K. et al. Localisation of transforming growth factor $\beta 1$ and $\beta 3$ mRNA transcripts in normal and fibrotic human lung. Thorax 56, 549-556 (2001).

57. Le, T. T. et al. Blockade of IL- 6 trans signaling attenuates pulmonary fibrosis. J. Immunol. 193, 3755-3768 (2014).

58. Moodley, Y. P. et al. Fibroblasts isolated from normal lungs and those with idiopathic pulmonary fibrosis differ in interleukin-6/gp130-mediated cell signaling and proliferation. Am. J. Pathol. 163, 345-354 (2003).

59. Chen, G. et al. Clinical and immunological features of severe and moderate coronavirus disease 2019. J. Clin. Invest. 130, 2620-2629 (2020).

60. Hendaus, M. A. \& Jomha, F. A. COVID-19 induced superimposed bacteria infection. J. Biomol. Struct. Dyn. https://doi.org/10.1080/07391102.2020.177 2110 (2020).

61. Hendaus, M. A., Jomha, F. A. \& Alhammadi, A. H. Virus-induced secondary bacterial infection: a concise review. Ther. Clin. Risk Manag. 11, 1265-1271 (2015)

62. Bharat, A. et al. Lung transplantation for patients with severe COVID-19. Sci. Transl. Med. 12, eabe4282 (2020).

63. Cui, S., Chen, S., Li, X., Liu, S. \& Wang, F. Prevalence of venous thromboembolism in patients with severe novel coronavirus pneumonia. J. Thromb. Haemost. 18, 1421-1424 (2020)

64. Klok, F. A. et al. Incidence of thrombotic complications in critically ill ICU patients with COVID-19. Thromb. Res. 191, 145-147 (2020).

65. Leonard-Lorant, I. et al. Acute pulmonary embolism in patients with COVID-19 at CT angiography and relationship to D-dimer levels. Radiology 296, E189-E191 (2020).

66. Middeldorp, S. et al. Incidence of venous thromboembolism in hospitalized patients with COVID-19. J. Thromb. Haemost. 18, 1995-2002 (2020).

67. Poissy, J. et al. Pulmonary embolism in patients with COVID-19: awareness of an increased prevalence. Circulation 142, 184-186 (2020).

68. Corrigan, D., Prucnal, C. \& Kabrhel, C. Pulmonary embolism: the diagnosis, risk-stratification, treatment and disposition of emergency department patients. Clin. Exp. Emerg. Med. 3, 117-125 (2016).

69. Lim, W. et al. Failure of anticoagulant thromboprophylaxis: risk factors in medical-surgical critically ill patients. Crit. Care Med. 43, 401-410 (2015).

70. Ackermann, M. et al. Pulmonary vascular endothelialitis, thrombosis, and angiogenesis in COVID-19. N. Engl. J. Med. 383, 120-128 (2020).

71. Lang, M. et al. Hypoxaemia related to COVID-19: vascular and perfusion abnormalities on dual-energy CT. Lancet Infect. Dis. 20, 1365-1366 (2020).

72. Bai, C. et al. Updated guidance on the management of COVID-19: from an American Thoracic Society/European Respiratory Society coordinated International Task Force (29 July 2020). Eur. Respir. Rev. 29, 200287 (2020).

73. Luks, A. M. \& Swenson, E. R. Pulse oximetry for monitoring patients with COVID-19 at home. Potential pitfalls and practical guidance. Ann. Am. Thorac. Soc. 17, 1040-1046 (2020).

74. Brigham, E. et al. The Johns Hopkins Post-Acute COVID-19 Team (PACT) a multidisciplinary, collaborative, ambulatory framework supporting COVID-19 survivors. Am. J. Med. https://doi.org/10.1016/j.amjmed. 2020.12.009 (2021)

75. Raghu, G. \& Wilson, K. C.COVID-19 interstitial pneumonia: monitoring the clinical course in survivors. Lancet Respir. Med. 8, 839-842 (2020).

76. George, P. M. et al. Respiratory follow-up of patients with COVID-19 pneumonia. Thorax 75, 1009-1016 (2020).

77. Myall, K. J. et al. Persistent post-COVID-19 inflammatory interstitial lung disease: an observational study of corticosteroid treatment. Ann. Am. Thorac. Soc. https://doi.org/10.1513/AnnalsATS.202008-1002OC (2021) 
78. Chang, Y. et al. Lung transplantation as a therapeutic option in acute respiratory distress syndrome. Transplantation 102, 829-837 (2018).

79. Wang, Q. et al. Lung transplantation in pulmonary fibrosis secondary to influenza A pneumonia. Ann. Thorac. Surg. 108, e233-e235 (2019).

80. Chen, J. et al. Lung transplantation for an ARDS patient post-COVID-19 infection. Chest 157, A453 (2020).

81. George, P. M., Wells, A. U. \& Jenkins, R. G. Pulmonary fibrosis and COVID-19: the potential role for antifibrotic therapy. Lancet Respir. Med. 8, 807-815 (2020).

82. Patell, R. et al. Post-discharge thrombosis and hemorrhage in patients with COVID-19. Blood 136, 1342-1346 (2020).

83. Roberts, L. N. et al. Post-discharge venous thromboembolism following hospital admission with COVID-19. Blood 136, 1347-1350 (2020).

84. Salisbury, R. et al. Incidence of symptomatic, image-confirmed venous thromboembolism following hospitalization for COVID-19 with 90-day follow-up. Blood Adv. 4, 6230-6239 (2020).

85. Engelen, M. et al. Incidence of venous thromboembolism in patients discharged after COVID-19 hospitalisation. Res. Pract. Thromb. Haemost. https://abstracts.isth.org/abstract/incidence-of-venous-thromboembolism-inpatients-discharged-after- covid-19-hospitalisation/ (2021).

86. Spyropoulos, A. C. et al. Scientific and Standardization Committee communication: clinical guidance on the diagnosis, prevention, and treatment of venous thromboembolism in hospitalized patients with COVID-19. J. Thromb. Haemost. 18, 1859-1865 (2020).

87. Mangion, K. et al. The Chief Scientist Office Cardiovascular and Pulmonary Imaging in SARS Coronavirus Disease-19 (CISCO-19) study. Cardiovasc. Res. 116, 2185-2196 (2020).

88. Pavoni, V. et al. Evaluation of coagulation function by rotation thromboelastometry in critically ill patients with severe COVID-19 pneumonia. J. Thromb. Thrombolysis 50, 281-286 (2020).

89. Chaudhary, R., Kreutz, R. P., Bliden, K. P., Tantry, U. S. \& Gurbel, P. A. Personalizing antithrombotic therapy in COVID-19: role of thromboelastography and thromboelastometry. Thromb. Haemost. 120, 1594-1596 (2020).

90. Connors, J. M. \& Levy, J. H. COVID-19 and its implications for thrombosis and anticoagulation. Blood 135, 2033-2040 (2020).

91. Varga, Z. et al. Endothelial cell infection and endotheliitis in COVID-19. Lancet 395, 1417-1418 (2020).

92. Goshua, G. et al. Endotheliopathy in COVID-19-associated coagulopathy: evidence from a single-centre, cross-sectional study. Lancet Haematol. 7, e575-e582 (2020).

93. Libby, P. \& Lüscher, T. COVID-19 is, in the end, an endothelial disease. Eur Heart J. 41, 3038-3044 (2020).

94. Ramlall, V. et al. Immune complement and coagulation dysfunction in adverse outcomes of SARS-CoV-2 infection. Nat. Med. 26, 1609-1615 (2020).

95. Skendros, P. et al. Complement and tissue factor-enriched neutrophil extracellular traps are key drivers in COVID-19 immunothrombosis. J. Clin. Invest. 130, 6151-6157 (2020).

96. Cugno, $M$. et al. Complement activation in patients with COVID-19: a novel therapeutic target. J. Allergy Clin. Immunol. 146, 215-217 (2020).

97. Hottz, E. D. et al. Platelet activation and platelet-monocyte aggregates formation trigger tissue factor expression in severe COVID-19 patients. Blood 136, 1330-1341 (2020).

98. Manne, B. K. et al. Platelet gene expression and function in COVID-19 patients. Blood 136, 1317-1329 (2020).

99. Barrett, T. J. et al. Platelet and vascular biomarkers associate with thrombosis and death in coronavirus disease. Circ. Res. https://doi.org/ 10.1161/CIRCRESAHA.120.317803 (2020).

100. Middleton, E. A. et al. Neutrophil extracellular traps (NETs) contribute to immunothrombosis in COVID-19 acute respiratory distress syndrome. Blood 136, 1169-1179 (2020).

101. Zuo, Y. et al. Neutrophil extracellular traps in COVID-19. JCI Insight 5, e138999 (2020).

102. Bikdeli, B. et al. Pharmacological agents targeting thromboinflammation in COVID-19: review and implications for future research. Thromb. Haemost. 120, 1004-1024 (2020)

103. Nougier, C. et al. Hypofibrinolytic state and high thrombin generation may play a major role in SARS-COV2 associated thrombosis. J. Thromb. Haemost. 18, 2215-2219 (2020).

104. Thachil, J. Hypoxia-an overlooked trigger for thrombosis in COVID-19 and other critically ill patients. J. Thromb. Haemost. 18, 3109-3110 (2020).

105. Merrill, J. T., Erkan, D., Winakur, J. \& James, J. A. Emerging evidence of a COVID-19 thrombotic syndrome has treatment implications. Nat. Rev. Rheumatol. 16, 581-589 (2020).

106. Bajaj, N. S. et al. Extended prophylaxis for venous thromboembolism after hospitalization for medical illness: a trial sequential and cumulative meta-analysis. PLoS Med. 16, e1002797 (2019).

107. Chiasakul, T. et al. Extended vs. standard-duration thromboprophylaxis in acutely ill medical patients: a systematic review and meta-analysis. Thromb. Res. 184, 58-61 (2019).
108. Bikdeli, B. et al. COVID-19 and thrombotic or thromboembolic disease: implications for prevention, antithrombotic therapy, and follow-up: JACC state-of-the-art review. J. Am. Coll. Cardiol. 75, 2950-2973 (2020).

109. Barnes, G. D. et al. Thromboembolism and anticoagulant therapy during the COVID-19 pandemic: interim clinical guidance from the anticoagulation forum. J. Thromb. Thrombolysis 50, 72-81 (2020).

110. COVID-19 and VTE/Anticoagulation: Frequently Asked Questions (American Society of Hematology, 2020); https://www.hematology.org/ covid-19/covid-19and- vte-anticoagulation

111. Moores, L. K. et al. Prevention, diagnosis, and treatment of VTE in patients with coronavirus disease 2019: CHEST Guideline and Expert Panel report. Chest 158, 1143-1163 (2020)

112. Jabri, A. et al. Incidence of stress cardiomyopathy during the coronavirus disease 2019 pandemic. JAMA Netw. Open 3, e2014780 (2020).

113. Puntmann, V. O. et al. Outcomes of cardiovascular magnetic resonance imaging in patients recently recovered from coronavirus disease 2019 (COVID-19). JAMA Cardiol. 5, 1265-1273 (2020).

114. Rajpal, S. et al. Cardiovascular magnetic resonance findings in competitive athletes recovering from COVID-19 infection. JAMA Cardiol. 6, 116-118 (2021).

115. Lindner, D. et al. Association of cardiac infection with SARS-CoV-2 in confirmed COVID-19 autopsy cases. JAMA Cardiol. 5, 1281-1285 (2020).

116. Gemayel, C., Pelliccia, A. \& Thompson, P. D. Arrhythmogenic right ventricular cardiomyopathy. J. Am. Coll. Cardiol. 38, 1773-1781 (2001)

117. Siripanthong, B. et al. Recognizing COVID-19-related myocarditis: the possible pathophysiology and proposed guideline for diagnosis and management. Heart Rhythm 17, 1463-1471 (2020).

118. Wu, Q. et al. Altered lipid metabolism in recovered SARS patients twelve years after infection. Sci. Rep. 7, 9110 (2017).

119. Liu, P. P., Blet, A., Smyth, D. \& Li, H. The science underlying COVID-19: implications for the cardiovascular system. Circulation 142, 68-78 (2020).

120. Lazzerini, P. E., Laghi-Pasini, F., Boutjdir, M. \& Capecchi, P. L. Cardioimmunology of arrhythmias: the role of autoimmune and inflammatory cardiac channelopathies. Nat. Rev. Immunol. 19, 63-64 (2019)

121. Agarwal, A. K., Garg, R., Ritch, A. \& Sarkar, P. Postural orthostatic tachycardia syndrome. Postgrad. Med. J. 83, 478-480 (2007).

122. Lau, S. T. et al. Tachycardia amongst subjects recovering from severe acute respiratory syndrome (SARS). Int. J. Cardiol. 100, 167-169 (2005).

123. Desai, A. D., Boursiquot, B. C., Melki, L. \& Wan, E. Y. Management of arrhythmias associated with COVID-19. Curr. Cardiol. Rep. 23, 2 (2020).

124. Hendren, N. S., Drazner, M. H., Bozkurt, B. \& Cooper, L. T. Jr. Description and proposed management of the acute COVID-19 cardiovascular syndrome. Circulation 141, 1903-1914 (2020).

125. Maron, B. J. et al. Eligibility and disqualification recommendations for competitive athletes with cardiovascular abnormalities: Task Force 3: hypertrophic cardiomyopathy, arrhythmogenic right ventricular cardiomyopathy and other cardiomyopathies, and myocarditis: a scientific statement from the American Heart Association and American College of Cardiology. J. Am. Coll. Cardiol. 66, 2362-2371 (2015).

126. Bozkurt, B., Kovacs, R. \& Harrington, B. Joint HFSA/ACC/AHA statement addresses concerns re: using RAAS antagonists in COVID-19. J. Card. Fail. 26, 370 (2020)

127. Lopes, R. D. et al. Effect of discontinuing vs continuing angiotensin-converting enzyme inhibitors and angiotensin II receptor blockers on days alive and out of the hospital in patients admitted with COVID-19: a randomized clinical trial. J. Am. Med. Assoc. 325, 254-264 (2021)

128. Vaduganathan, M. et al. Renin-angiotensin-aldosterone system inhibitors in patients with COVID-19. N. Engl. J. Med. 382, 1653-1659 (2020).

129. Guzik, T. J. et al. COVID-19 and the cardiovascular system: implications for risk assessment, diagnosis, and treatment options. Cardiovasc Res. 116, 1666-1687 (2020)

130. Rey, J. R. et al. Heart failure in COVID-19 patients: prevalence, incidence and prognostic implications. Eur. J. Heart Fail. 22, 2205-2215 (2020).

131. Raj, S. R. et al. Propranolol decreases tachycardia and improves symptoms in the postural tachycardia syndrome: less is more. Circulation 120, 725-734 (2009).

132. Kociol, R. D. et al. Recognition and initial management of fulminant myocarditis: a scientific statement from the American Heart Association. Circulation 141, e69-e92 (2020).

133. Fauci, A. International AIDS conference. YouTube https://www.youtube. $\mathrm{com} /$ watch? $\mathrm{v}=\mathrm{UMmT} 48 \mathrm{ICO}$ us \&feature $=$ emb_logo $(2020)$.

134. Nordvig, A. S. et al. Potential neurological manifestations of COVID-19. Neurol. Clin. Pract. https://doi.org/10.1212/CPJ.0000000000000897 (2020).

135. Belvis, R. Headaches during COVID-19: my clinical case and review of the literature. Headache 60, 1422-1426 (2020).

136. Arca, K. N. \& Starling, A. J.Treatment-refractory headache in the setting of COVID-19 pneumonia: migraine or meningoencephalitis? Case report. SN Compr. Clin. Med. 2, 1200-1203 (2020). 
137. Bolay, H., Gül, A. \& Baykan, B. COVID-19 is a real headache! Headache https://doi.org/10.1111/head.13856 (2020).

138. Pozo-Rosich, P. Headache \& COVID-19: a short-term challenge with long-term insights. In Proc. AHSAM 2020 Virtual Annual Scientific Meeting (Infomedica, 2020); https://www.ahshighlights.com/ summaries-podcasts/ article/ headache-covid-19-a-short- term-challenge-with- long-term-insight

139. Heneka, M. T., Golenbock, D., Latz, E., Morgan, D. \& Brown, R. Immediate and long-term consequences of COVID-19 infections for the development of neurological disease. Alzheimers Res. Ther. 12, 69 (2020).

140. Ritchie, K., Chan, D. \& Watermeyer, T. The cognitive consequences of the COVID-19 epidemic: collateral damage? Brain Commun. 2, fcaa069 (2020)

141. Kaseda, E. T. \& Levine, A. J. Post-traumatic stress disorder: a differential diagnostic consideration for COVID-19 survivors. Clin. Neuropsychol. 34, 1498-1514 (2020)

142. Postolache, T. T., Benros, M. E. \& Brenner, L. A. Targetable biological mechanisms implicated in emergent psychiatric conditions associated with SARS-CoV-2 infection. JAMA Psychiatry https://doi.org/10.1001/ jamapsychiatry.2020.2795 (2020).

143. Mazza, M. G. et al. Anxiety and depression in COVID-19 survivors: role of inflammatory and clinical predictors. Brain Behav. Immun. 89, 594-600 (2020).

144. Rogers, J. P. et al. Psychiatric and neuropsychiatric presentations associated with severe coronavirus infections: a systematic review and meta-analysis with comparison to the COVID-19 pandemic. Lancet Psychiatry 7, 611-627 (2020).

145. Taquet, M., Luciano, S., Geddes, J. R. \& Harrison, P. J. Bidirectional associations between COVID-19 and psychiatric disorder: retrospective cohort studies of 62354 COVID-19 cases in the USA. Lancet Psychiatry 8, 130-140 (2021).

146. Trejo-Gabriel-Galán, J. M. Stroke as a complication and prognostic factor of COVID-19. Neurologia 35, 318-322 (2020)

147. Parauda, S. C. et al. Posterior reversible encephalopathy syndrome in patients with COVID-19. J. Neurol. Sci. 416, 117019 (2020).

148. Ellul, M. A. et al. Neurological associations of COVID-19. Lancet Neurol. 19, 767-783 (2020).

149. Paterson, R. W. et al. The emerging spectrum of COVID-19 neurology: clinical, radiological and laboratory findings. Brain 143, 3104-3120 (2020).

150. Tankisi, H. et al. Critical illness myopathy as a consequence of COVID-19 infection. Clin. Neurophysiol. 131, 1931-1932 (2020).

151. Muccioli, L. et al. COVID-19-associated encephalopathy and cytokine-mediated neuroinflammation. Ann. Neurol. 88, 860-861 (2020).

152. Pilotto, A., Padovani, A. \& ENCOVID-BIO Network. Reply to the letter "COVID-19-associated encephalopathy and cytokine-mediated neuroinflammation". Ann, Neurol. 88, 861-862 (2020).

153. South, K. et al. Preceding infection and risk of stroke: an old concept revived by the COVID-19 pandemic. Int J. Stroke 15, 722-732 (2020).

154. Desforges, M., Le Coupanec, A., Stodola, J. K., Meessen-Pinard, M. \& Talbot, P. J. Human coronaviruses: viral and cellular factors involved in neuroinvasiveness and neuropathogenesis. Virus Res. 194, 145-158 (2014)

155. Romero-Sánchez, C. M. et al. Neurologic manifestations in hospitalized patients with COVID-19: the ALBACOVID registry. Neurology 95, e1060-e1070 (2020).

156. Reichard, R. R. et al. Neuropathology of COVID-19: a spectrum of vascular and acute disseminated encephalomyelitis (ADEM)-like pathology. Acta Neuropathol. 140, 1-6 (2020).

157. Bortolato, B., Carvalho, A. F., Soczynska, J. K., Perini, G. I. \& McIntyre, R. $\mathrm{S}$. The involvement of TNF- $\alpha$ in cognitive dysfunction associated with major depressive disorder: an opportunity for domain specific treatments. Curr. Neuropharmacol. 13, 558-576 (2015).

158. Aiello, A. et al. Immunosenescence and its hallmarks: how to oppose aging strategically? A review of potential options for therapeutic intervention. Front. Immunol. 10, 2247 (2019).

159. Perrin, R. et al. Into the looking glass: post-viral syndrome post COVID-19. Med. Hypotheses 144, 110055 (2020).

160. Morbini, P. et al. Ultrastructural evidence of direct viral damage to the olfactory complex in patients testing positive for SARS-CoV-2. JAMA Otolaryngol. Head Neck Surg. https://doi.org/10.1001/jamaoto.2020.2366 (2020).

161. Ameres, M. et al. Association of neuronal injury blood marker neurofilament light chain with mild-to-moderate COVID-19. J. Neurol. 267, 3476-3478 (2020)

162. Kanberg, N. et al. Neurochemical evidence of astrocytic and neuronal injury commonly found in COVID-19. Neurology https://doi.org/10.1212/ WNL.0000000000010111 (2020).

163. Novak, P. Post COVID-19 syndrome associated with orthostatic cerebral hypoperfusion syndrome, small fiber neuropathy and benefit of immunotherapy: a case report. eNeurologicalSci 21, 100276 (2020).

164. Miglis, M. G., Goodman, B. P., Chémali, K. R. \& Stiles, L. Re: 'Post-COVID-19 chronic symptoms' by Davido et al. Clin. Microbiol. Infect. https://doi.org/10.1016/j.cmi.2020.08.028 (2020).
165. Sakusic, A. \& Rabinstein, A. A. Cognitive outcomes after critical illness. Curr. Opin. Crit. Care 24, 410-414 (2018).

166. Do, T. P. et al. Red and orange flags for secondary headaches in clinical practice: SNNOOP10 list. Neurology 92, 134-144 (2019).

167. Robbins-Juarez, S. Y. et al. Outcomes for patients with COVID-19 and acute kidney injury: a systematic review and meta-analysis. Kidney Int. Rep. 5, 1149-1160 (2020)

168. Cummings, M. J. et al. Epidemiology, clinical course, and outcomes of critically ill adults with COVID-19 in New York City: a prospective cohort study. Lancet 395, 1763-1770 (2020).

169. Gupta, S. et al. Factors associated with death in critically ill patients with coronavirus disease 2019 in the US. JAMA Intern. Med. 180, 1-12 (2020)

170. Stevens, J. S. et al. High rate of renal recovery in survivors of COVID-19 associated acute renal failure requiring renal replacement therapy. PLoS ONE 15, e0244131 (2020).

171. Wilbers, T. J. \& Koning, M. V. Renal replacement therapy in critically ill patients with COVID-19: a retrospective study investigating mortality, renal recovery and filter lifetime. J. Crit. Care 60, 103-105 (2020).

172. Su, H. et al. Renal histopathological analysis of 26 postmortem findings of patients with COVID-19 in China. Kidney Int. 98, 219-227 (2020).

173. Kudose, S. et al. Kidney biopsy findings in patients with COVID-19. J. Am. Soc. Nephrol. 31, 1959-1968 (2020).

174. Sharma, P. et al. COVID-19-associated kidney injury: a case series of kidney biopsy findings. J. Am. Soc. Nephrol. 31, 1948-1958 (2020).

175. Golmai, P. et al. Histopathologic and ultrastructural findings in postmortem kidney biopsy material in 12 patients with AKI and COVID-19. J. Am. Soc. Nephrol. 31, 1944-1947 (2020).

176. Santoriello, D. et al. Postmortem kidney pathology findings in patients with COVID-19. J. Am. Soc. Nephrol. 31, 2158-2167 (2020).

177. Velez, J. C. Q., Caza, T. \& Larsen, C. P. COVAN is the new HIVAN: the re-emergence of collapsing glomerulopathy with COVID-19. Nat. Rev. Nephrol. 16, 565-567 (2020).

178. Peleg, Y. et al. Acute kidney injury due to collapsing glomerulopathy following COVID-19 Infection. Kidney Int. Rep. 5, 940-945 (2020).

179. Jhaveri, K. D. et al. Thrombotic microangiopathy in a patient with COVID-19. Kidney Int. 98, 509-512 (2020)

180. Meier, P., Bonfils, R. M., Vogt, B., Burnand, B. \& Burnier, M. Referral patterns and outcomes in noncritically ill patients with hospital-acquired acute kidney injury. Clin. J. Am. Soc. Nephrol. 6, 2215-2225 (2011).

181. Harel, Z. et al. Nephrologist follow-up improves all-cause mortality of severe acute kidney injury survivors. Kidney Int. 83, 901-908 (2013).

182. Suwanwongse, K. \& Shabarek, N. Newly diagnosed diabetes mellitus, DKA and COVID-19: causality or coincidence? A report of three cases. J. Med. Virol. https://doi.org/10.1002/jmv.26339 (2020).

183. Rubino, F. et al. New-onset diabetes in COVID-19. N. Engl. J. Med. 383, 789-790 (2020).

184. Ruggeri, R. M., Campenni, A., Siracusa, M., Frazzetto, G. \& Gullo, D.Subacute thyroiditis in a patient infected with SARS-COV-2: an endocrine complication linked to the COVID-19 pandemic. Hormones (Athens) 20, 219-221 (2021).

185. Brancatella, A. et al. Subacute thyroiditis after SARS-COV-2 infection. J. Clin. Endocrinol. Metab. 105, dgaa276 (2020).

186. Tee, L. Y., Hajanto, S. \& Rosario, B. H. COVID-19 complicated by Hashimoto's thyroiditis. Singapore Med. J. https://doi.org/10.11622/smedj. 2018150 (2020).

187. Mateu-Salat, M., Urgell, E. \& Chico, A.SARS-COV-2 as a trigger for autoimmune disease: report of two cases of Graves' disease after COVID-19. J. Endocrinol. Invest. 43, 1527-1528 (2020).

188. Gentile, S., Strollo, F., Mambro, A. \& Ceriello, A.COVID-19, ketoacidosis and new-onset diabetes: are there possible cause and effect relationships among them? Diabetes Obes. Metab. 22, 2507-2508 (2020).

189. Yang, J. K., Lin, S. S., Ji, X. J. \& Guo, L. M. Binding of SARS coronavirus to its receptor damages islets and causes acute diabetes. Acta Diabetol. 47, 193-199 (2010).

190. Salvio, G. et al. Bone metabolism in SARS-CoV-2 disease: possible osteoimmunology and gender implications. Clin. Rev. Bone Miner. Metab. https://doi.org/10.1007/s12018-020-09274-3 (2020).

191. DiMeglio, L. A., Evans-Molina, C. \& Oram, R. A. Type 1 diabetes. Lancet 391, 2449-2462 (2018)

192. Cheung, K. S. et al. Gastrointestinal manifestations of SARS-CoV-2 infection and virus load in fecal samples from a Hong Kong cohort: systematic review and meta-analysis. Gastroenterology 159, 81-95 (2020).

193. Wu, Y. et al. Prolonged presence of SARS-CoV-2 viral RNA in faecal samples. Lancet Gastroenterol. Hepatol. 5, 434-435 (2020).

194. Xiao, F. et al. Evidence for gastrointestinal infection of SARS-CoV-2. Gastroenterology 158, 1831-1833.e3 (2020).

195. Xu, Y. et al. Characteristics of pediatric SARS-CoV-2 infection and potential evidence for persistent fecal viral shedding. Nat. Med. 26, 502-505 (2020). 
196. Zuo, T. et al. Alterations in gut microbiota of patients with COVID-19 during time of hospitalization. Gastroenterology 159, 944-955.e8 (2020)

197. Donati Zeppa, S., Agostini, D., Piccoli, G., Stocchi, V. \& Sestili, P.Gut microbiota status in COVID-19: an unrecognized player? Front. Cell. Infect. Microbiol. 10, 576551 (2020).

198. Bradley, K. C. et al. Microbiota-driven tonic interferon signals in lung stromal cells protect from influenza virus infection. Cell Rep. 28, 245-256. e4 (2019).

199. Miquel, S. et al. Faecalibacterium prausnitzii and human intestinal health. Curr. Opin. Microbiol. 16, 255-261 (2013).

200. Freeman, E. E. et al. The spectrum of COVID-19-associated dermatologic manifestations: an international registry of 716 patients from 31 countries. J. Am. Acad. Dermatol. 83, 1118-1129 (2020).

201. Mirza, F. N., Malik, A. A., Omer, S. B. \& Sethi, A. Dermatologic manifestations of COVID-19: a comprehensive systematic review. Int. J. Dermatol. https://doi.org/10.1111/ijd.15168 (2020).

202. Genovese, G., Moltrasio, C., Berti, E. \& Marzano, A. V.Skin manifestations associated with COVID-19: current knowledge and future perspectives. Dermatology 237, 1-12 (2020).

203. Information for Healthcare Providers about Multisystem Inflammatory Syndrome in Children (MIS-C) (Centers for Disease Control and Prevention, 2020); https://www.cdc.gov/mis-c/hcp/

204. Multisystem Inflammatory Syndrome in Children and Adolescents with COVID-19 (World Health Organization, 2020); https://www.who.int/ publications/i/item/ multisystem-inflammatory- syndrome-in-childrenand-adolescents- with-covid-19

205. Jiang, L. et al. COVID-19 and multisystem inflammatory syndrome in children and adolescents. Lancet Infect. Dis. 20, e276-e288 (2020).

206. Henderson, L. A. et al. American College of Rheumatology clinical guidance for multisystem inflammatory syndrome in children associated with SARS-CoV-2 and hyperinflammation in pediatric COVID-19: version 1. Arthritis Rheumatol. 72, 1791-1805 (2020).

207. Paediatric Multisystem Inflammatory Syndrome Temporally Associated with COVID-19 (PIMS)_Guidance for Clinicians (Royal College of Paediatrics and Child Health, 2020); https://www.rcpch.ac.uk/ resources/paediatricmultisystem-inflammatory- syndrome-temporally- associated-covid19-pims-guidance

208. Rowley, A. H. Understanding SARS-CoV-2-related multisystem inflammatory syndrome in children. Nat. Rev. Immunol. 20, 453-454 (2020).

209. Schupper, A. J., Yaeger, K. A. \& Morgenstern, P. F. Neurological manifestations of pediatric multi-system inflammatory syndrome potentially associated with COVID-19. Childs Nerv. Syst. 36, 1579-1580 (2020).

210. Lin, J. E. et al. Neurological issues in children with COVID-19. Neurosci. Lett. 743, 135567 (2021)

211. Nakra, N. A., Blumberg, D. A., Herrera-Guerra, A. \& Lakshminrusimha, S. Multi-system inflammatory syndrome in children (MIS-C) following SARS-CoV-2 infection: review of clinical presentation, hypothetical pathogenesis, and proposed management. Children (Basel) 7, 69 (2020).

212. McCrindle, B. W. et al. Diagnosis, treatment, and long-term management of Kawasaki disease: a scientific statement for health professionals from the American Heart Association. Circulation 135, e927-e999 (2017).

213. $\mathrm{Gu}$, T. et al. Characteristics associated with racial/ethnic disparities in COVID-19 outcomes in an academic health care system. JAMA Netw. Open 3, e2025197 (2020)

214. Yancy, C. W. COVID-19 and African Americans. J. Am. Med. Assoc. 323, 1891-1892 (2020).

215. Mackey, K. et al. Racial and ethnic disparities in COVID-19-related infections, hospitalizations, and deaths: a systematic review. Ann. Int. Med. https://doi.org/10.7326/M20-6306 (2020).

216. Webb Hooper, M., Nápoles, A. M. \& Pérez-Stable, E. J. COVID-19 and racial/ethnic disparities. J. Am. Med. Assoc. 323, 2466-2467 (2020).

217. Bunyavanich, S., Grant, C. \& Vicencio, A. Racial/ethnic variation in nasal gene expression of transmembrane serine protease 2 (TMPRSS2). J. Am. Med. Assoc. 324, 1567-1568 (2020).

218. Chowkwanyun, M. \& Reed, A. L. Racial health disparities and COVID-19caution and context. N. Engl. J. Med. 383, 201-203 (2020).

219. Brugliera, L. et al. Nutritional management of COVID-19 patients in a rehabilitation unit. Eur. J. Clin. Nutr. 74, 860-863 (2020).

220. Caccialanza, R. et al. Early nutritional supplementation in non-critically ill patients hospitalized for the 2019 novel coronavirus disease (COVID-19): rationale and feasibility of a shared pragmatic protocol. Nutrition $\mathbf{7 4}$, 110835 (2020)

221. Sosnowski, K., Lin, F., Mitchell, M. L. \& White, H. Early rehabilitation in the intensive care unit: an integrative literature review. Aust. Crit. Care $\mathbf{2 8}$ 216-225 (2015).

222. Simpson, R. \& Robinson, L. Rehabilitation after critical illness in people with COVID-19 infection. Am. J. Phys. Med. Rehabil. 99, 470-474 (2020).
223. Masiero, S., Zampieri, D. \& Del Felice, A. The place of early rehabilitation in intensive care unit for COVID-19. Am. J. Phys. Med. Rehabil. 99 677-678 (2020).

224. Puchner, B. et al. Beneficial effects of multi-disciplinary rehabilitation in post-acute COVID-19-an observational cohort study. Eur. J. Phys. Rehabil. Med. https://doi.org/10.23736/S1973-9087.21.06549-7 (2021).

225. Rubin, R. As their numbers grow, COVID-19 "long haulers" stump experts. J. Am. Med. Assoc. 324, 1381-1383 (2020).

226. Long COVID: let patients help define long-lasting COVID symptoms. Nature 586, 170 (2020).

\section{Acknowledgements}

We acknowledge J. Der-Nigoghossian and BioRender for design support for the figures. D.E.F. was supported in part by National Institutes of Health grant K23 DK111847 and by Department of Defense funding PR181960. M.V.M. was supported by an institutional grant from the National Institutes of Health/National Heart, Lung, and Blood Institute to Columbia University Irving Medical Center (T32 HL007854). S.M. was supported by National Institute of Diabetes and Digestive and Kidney Diseases grants R01-DK114893, R01-MD014161 and U01-DK116066, as well as National Science Foundation grant 2032726. A.S.N. was supported by National Institute of Neurological Disorders and Stroke grant T32 NS007153-36 and National Institute on Aging grant P30 AG066462-01. E.Y.W. was supported by NIH R01 HL152236 and R03 HL146881, the Esther Aboodi Endowed Professorship at Columbia University, the Foundation for Gender-Specific Medicine, the Louis V. Gerstner, Jr. Scholars Program and the Wu Family Research Fund. The funders had no role in the design or conduct of the study; collection, management, analysis or interpretation of the data; preparation, review or approval of the manuscript; or decision to submit the manuscript for publication. The content is solely the responsibility of the authors and does not necessarily represent the official views of the funding agencies.

\section{Competing interests}

D.A. is founder, director and chair of the advisory board of Forkhead Therapeutics. B.B. reports being a consulting expert, on behalf of the plaintiff, for litigation related to two specific brand models of inferior vena cava filter. D.B. receives research support from ALung Technologies and is on the medical advisory boards for Baxter, Abiomed, Xenios and Hemovent. T.K.C. reports research support (institutional and personal) from AstraZeneca, Alexion, Bayer, Bristol-Myers Squibb/ER Squibb and Sons, Cerulean, Eisai, Foundation Medicine, Exelixis, Ipsen, Tracon, Genentech, Roche, Roche Products, F. Hoffmann-La Roche, GlaxoSmithKline, Lilly, Merck, Novartis, Peloton, Pfizer, Prometheus Laboratories, Corvus, Calithera, Analysis Group, Sanofi/Aventis and Takeda; honoraria from AstraZeneca, Alexion, Sanofi/Aventis, Bayer, Bristol-Myers Squibb/ER Squibb and Sons, Cerulean, Eisai, Foundation Medicine, Exelixis, Genentech, Roche, Roche Products, F. Hoffmann-La Roche, GlaxoSmithKline, Merck, Novartis, Peloton, Pfizer, EMD Serono, Prometheus Laboratories, Corvus, Ipsen, UpToDate, NCCN, Analysis Group, Michael J. Hennessy (MJH) Associates (a healthcare communications company with several brands such as OncLive, PeerView and PER), Research to Practice, Lpath, Kidney Cancer, Clinical Care Options, PlatformQ, Navinata Health, Harborside Press, the American Society of Medical Oncology, the New England Journal of Medicine, Lancet Oncology, Heron Therapeutics and Lilly Oncology; a consultant or advisory role for AstraZeneca, Alexion, Sanofi/Aventis, Bayer, Bristol-Myers Squibb/ ER Squibb and Sons, Cerulean, Eisai, Foundation Medicine, Exelixis, Genentech, Heron Therapeutics, Lilly, Roche, GlaxoSmithKline, Merck, Novartis, Peloton, Pfizer, EMD Serono, Prometheus Laboratories, Corvus, Ipsen, UpToDate, NCCN, Analysis Group, Pionyr, Tempest and Lilly Ventures; stock ownership in Pionyr and Tempest; and medical writing and editorial assistance support from communications companies funded by pharmaceutical companies (ClinicalThinking, Envision Pharma Group, Fishawack Group of Companies, Health Interactions, Parexel, Oxford PharmaGenesis and others). J.M.C. reports a consultant or advisory role for Abbott Vascular, Bristol-Myers Squibb, Portola and Takeda, as well as research support (institutional) from CSL Behring. M.S.V.E. reports receiving royalties from UpToDate for chapters on stroke and COVID-19. A.G. received payment from the Arnold \& Porter law firm for work related to the Sanofi clopidogrel litigation and from the Ben C. Martin law firm for work related to the Cook inferior vena cava filter litigation; received consulting fees from Edward Lifesciences; and holds equity in the healthcare telecardiology startup Heartbeat Health. D.W.L. is chair of the scientific advisory board for Applied Therapeutics, which licenses Columbia University technology unrelated to COVID-19 or COVID-19-related therapies.

\section{Additional information}

\section{Correspondence should be addressed to K.S. or E.Y.W.}

Peer review information Nature Medicine thanks Andrew Chan, Eike Nagel and the other, anonymous, reviewer(s) for their contribution to the peer review of their work. Joao Monteiro was the primary editor on this article and managed its editorial process and peer review in collaboration with the rest of the editorial team.

Reprints and permissions information is available at www.nature.com/reprints. Publisher's note Springer Nature remains neutral with regard to jurisdictional claims in published maps and institutional affiliations.

(c) The Author(s), under exclusive licence to Springer Nature America, Inc. 2021 